\title{
Lucky spectroscopy, an equivalent technique to lucky imaging
}

\section{Spatially resolved intermediate-resolution blue-violet spectroscopy of 19 close massive binaries using the William Herschel Telescope}

\author{
J. Maíz Apellániz ${ }^{1}$, R. H. Barbá ${ }^{2}$ C. Fariña ${ }^{3,4}$, A. Sota ${ }^{5}$, M. Pantaleoni González ${ }^{1,6}$, G. Holgado ${ }^{1}$, \\ I. Negueruela ${ }^{7}$, and S. Simón-Díaz ${ }^{3,8}$
}

\begin{abstract}
${ }^{1}$ Centro de Astrobiología, CSIC-INTA. Campus ESAC, Camino bajo del castillo s/n, 28692 Vill. de la Cañada, Madrid, Spain e-mail: jmaiz@cab.inta-csic.es

2 Departamento de Astronomía. Universidad de La Serena, Av. Cisternas 1200 Norte, La Serena, Chile

3 Instituto de Astrofísica de Canarias, 38200 La Laguna, Tenerife, Spain

4 Isaac Newton Group of Telescopes, Apartado de correos 321, 38700 Santa Cruz de La Palma, La Palma, Spain

5 Instituto de Astrofísica de Andalucía-CSIC, Glorieta de la Astronomía s/n, 18008 Granada, Spain

6 Departamento de Astrofísica y Física de la Atmósfera. Universidad Complutense de Madrid, 28040 Madrid, Spain

7 Departamento de Física. Ingeniería de Sistemas y Teoría de la Señal. Escuela Politécnica Superior. Universidad de Alicante, Carretera San Vicente del Raspeig s/n, 03690 San Vicente del Raspeig, Alicante, Spain

8 Departamento de Astrofísica. Universidad de La Laguna, 38205 La Laguna, Tenerife, Spain
\end{abstract}

Received 21 September 2020 / Accepted 22 November 2020

\begin{abstract}
Context. Many massive stars have nearby companions. These hamper a characterization of massive stars through spectroscopy. Aims. We continue to obtain spatially resolved spectroscopy of close massive visual binaries to derive their spectral types.

Methods. We used the lucky spectroscopy technique to obtain a large number of short long-slit spectroscopic exposures of 19 close visual binaries under good seeing conditions. We selected those with the best characteristics, extracted the spectra using multipleprofile fitting, and combined the results to derive spatially separated spectra. The results were analyzed in combination with data from lucky imaging, regular intermediate-resolution single-order spectroscopy, and échelle high-resolution spectroscopy.

Results. The new application of lucky spectroscopy has allowed us (among other results) to [a] spatially disentangle two O stars (FN CMa B and 6 Cas B) with brighter BA supergiant companions for the first time; [b] determine that two B stars ( $\alpha$ Sco B and HD 164492 B) with close and more massive companions are fast rotators (in the second case, solving a case of mistaken identity); [c] extend the technique to cases with extreme magnitude differences (the previous two cases plus CS Cam A,B), shorter separations (HD $193443 \mathrm{~A}, \mathrm{~B}$ ), and fainter primary magnitudes down to $B=11$ (HD $219460 \mathrm{~A}, \mathrm{~B}$ ); [d] spatially disentangle the spectra of stars with companions as diverse as an A supergiant (6 Cas A), a Wolf-Rayet star (HD $219460 \mathrm{~B}=\mathrm{WR}$ 157), and an M supergiant ( $\alpha$ Sco A); [e] discover the unexpected identity of some targets such as two previously unknown bright O stars (HD $51756 \mathrm{~B}$ and $\mathrm{BD}+60544$ ) and a new member of the rare OC category (HD $8768 \mathrm{~A}$ ); and [f] identify and classify (in some cases for the first time) which of the components of four visual binaries ( $\sigma$ Ori, HD 219460, HD 194649, and HD 191 201) is a double-lined spectroscopic binary. For another seven systems (FN CMa, $\sigma$ Sco, HD 51 756, HD 218 195, HD 17 520, HD 24 431, and HD 164 492), we detect signs of spectroscopic binarity using high-spectral-resolution spectroscopy. We also determine the limits of the technique.
\end{abstract}

Key words. binaries: spectroscopic - binaries: visual - methods: data analysis - stars: early-type - stars: massive techniques: spectroscopic

\section{Introduction}

Massive stars are born in the company of others, but their multiplicity is not always easy to detect or characterize. The main complication lies in the wide range of periods, from $\sim 1 \mathrm{~d}$ orbits to systems that complete just one revolution or a few revolutions during the lifetimes of the stars. For a complete account of massive-star multiplicity, we therefore need to use different techniques because long-period systems are better studied with techniques that use high spatial resolution and short-period systems are more easily understood with techniques that use high spectral resolution. In other words, the first type are usually studied as visual binaries (or multiples), and the second type as spectroscopic binaries (or multiples) (Mason et al. 1998). In multiplicity studies, it should be clear which type (one, the other, or both) we refer to. Otherwise, confusion is likely to arise and incorrect results will be obtained.

The first golden era of spectroscopic surveys of OB stars lasted from the 1950s to the 1970s. Morgan et al. (1955), Hiltner (1956), Lesh (1968), Hiltner et al. (1969), Walborn (1972, 1973), and Garrison et al. (1977) are probably the best examples of the results of that period. Interest in such surveys waned in the following two decades (with some exceptions), but regained strength in the 2000s with new technology and telescopes that significantly increased observing efficiency and with the need to address scientific issues such as the ubiquitous multiplicity described above. As part of this renewed interest, we have been conducting different types of surveys for some time now that have the ultimate goal of characterizing massive stars and, more specifically, their multiplicity. With the Galactic O-Star 
Spectroscopic Survey (GOSSS, Maíz Apellániz et al. 2011) we are identifying and producing spectral classifications for $\mathrm{O}$ stars (and other massive stars) in the Galactic neighborhood with intermediate-resolution blue-violet spectroscopy. GOSSS has resulted in three major papers: Sota et al. (2011; GOSSS I), Sota et al. (2014; GOSSS II), and Maíz Apellániz et al. (2016; GOSSS III), with a fourth paper in the series to be submitted soon. We have also collected a library of high-resolution spectroscopy of massive stars (LiLiMaRlin, Maíz Apellániz et al. 2019a) from public and private observations. In the northern hemisphere, we use this to study the spectroscopic multiplicity of O stars (MONOS project) of which the first paper appeared as Maíz Apellániz et al. (2019b; MONOS I), and a second paper will be submitted soon. In a parallel effort, GOSSS also serves as a source of information for IACOB (Simón-Díaz et al. 2015b), a long-term project aimed at extending the empirical characterization of Galactic O (and B) stars further using quantitative highresolution spectroscopy (see, e.g., Simón-Díaz et al. 2014, 2017; Holgado et al. 2018, 2020). As for high spatial resolution observations, we have observed a large number of systems with lucky imaging using AstraLux (Maíz Apellániz 2010 and MONOS I).

The surveys in the previous paragraph use either spectroscopic techniques or techniques with high spatial resolution, but they do not combine them. In some cases such as hierarchical systems composed of a short-period spectroscopic binary and a close visual companion, the relation of the three stars may therefore be unclear. Even the assignment of a precise spectral type to each may be in question (for an example, $\sigma$ Ori, see Simón-Díaz et al. 2015a and Sect. 3.6). Combining the two techniques (i.e., spectroscopy with high spatial resolution) addresses this problem. We did this on the one hand using Space Telescope Imaging Spectrograph (STIS) spectroscopy with the Hubble Space Telescope (HST, Maíz Apellániz \& Barbá 2020, from now on STIS I) or applying the novel technique of lucky spectroscopy (Maíz Apellániz et al. 2018, from now on LS I), which we developed in LS I. Lucky spectroscopy is the extension of lucky imaging (Law et al. 2006) to spectroscopy: we obtain a large number of short long-slit spectroscopic exposures under good seeing conditions, select those with the best characteristics, and combine them to derive spatially resolved spectra of two or more closely separated point sources aligned with the slit.

In this paper we continue our analysis of massive close binaries with lucky spectroscopy that we started in LS I. In the next section we describe the main data in the paper, our new lucky spectroscopy observations for 19 close visual multiple systems, and their relation with the previous data. We also describe the complementary data we used to analyze these systems in the form of lucky imaging and other spectroscopy. In Sect. 3 we present the results for our sample. We summarize our results and briefly present our future plans.

\section{Data and methods}

\subsection{Lucky spectroscopy}

In LS I we presented lucky spectroscopy results for five bright (primaries with $B$ magnitudes of 2-4) close visual binaries whose primaries have spectral subtypes between 07 and B0.7. The original data were obtained in September 2017 (although several tests had been performed previously, see below for an example) with the ISIS spectrograph at the William Herschel Telescope (WHT). The success of the technique prompted us to attempt the separation of additional systems in 2018 and 2019. We had also obtained time for further observations in 2020, but they did not take place due to scheduling changes at the WHT. In addition to the obvious scientific interest of studying more objects to obtain knowledge of the members of the larger sample, we were interested in exploring four avenues:

1. Repeatability of the results, including new epochs for systems that contain spectroscopic binaries that may be observed at a more favourable orbital phase.

2. Coverage of the separation-magnitude difference $(d-\Delta m)$ plane to test the limitations of the technique and comparison with other techniques (STIS I).

3. Exploration of systems with spectral types significantly different from the relatively narrow scope of LS I.

4. Extension of the technique to systems with fainter primaries.

In this paper we present our results for 19 close visual binaries observed with lucky spectroscopy (one from the previous sample and 18 new binaries, Table 1), which were selected with these points in mind. The setup and technique we used are the same as in LS I with one difference. In that paper we used the standard CCD for the blue arm of ISIS, an EEV12 array of $2048 \times 4096$ $13.5 \mu \mathrm{m}$ pixels. Here we used this same CCD as the default setup, but we also tried the alternative QUCAM3 CCD for the faint range of our sample. QUCAM3 is an electron-multiplying CCD with $1024 \times 102413 \mu$ mpixels with a fast mode that has very little readout noise (which we did not use) and a standard slow mode in which it operates as a normal CCD (which we used). The great advantage of QUCAM3 over EEV12 is that it is a fulltransfer device that allows a quick transfer (20 milliseconds) of the accumulated charge (the image) into a temporary on-chip storage to be efectivelly read in $0.66 \mathrm{~s}$ (for the detector setup used for these data) while the detector still actively acquires data ${ }^{1}$. For one-second exposures, the dead time is therefore just $\sim 2 \%$, while for EEV12, the corresponding dead time is $\sim 14 \mathrm{~s}$, introducing a factor of nearly 15 in the efficiency in favour of QUCAM3. A second less important advantage of QUCAM3 is the slightly better pixel scale $\left(0^{\prime \prime} 19\right.$ pixels vs. $00^{\prime \prime} 20$ pixels). The main disadvantage of QUCAM3 arises from its smaller number of pixels: with EEV12 we can cover the 3900-5500 A range with a single grating position, while with QUCAM 3 we need three grating positions to cover just the $3900-5100 \AA$ range. These characteristics translate into different observing sequences for the two setups. For EEV12 we have just three steps:

1. Acquire the target and place the slit at the correct position angle (typically $4 \mathrm{~m}$ ).

2. Obtain 100 exposures of $1.0 \mathrm{~s}$ each in a single grating position $(25 \mathrm{~m})$.

3. Perform an arc $(1 \mathrm{~m})$.

This yields $30 \mathrm{~min}$ per object and $100 \mathrm{~s}$ of total exposure time, of which we typically finally used $10-50 \mathrm{~s}$. For QUCAM3 we have to follow seven steps:

1. Acquire the target and place the slit at the correct position angle (typically $4 \mathrm{~m}$ ).

2. Obtain 500 exposures of $1.0 \mathrm{~s}$ each in the first grating position $(8.5 \mathrm{~m})$.

3. Perform an arc and change to the second grating position $(1.5 \mathrm{~m})$.

4. Obtain 500 exposures of $1.0 \mathrm{~s}$ each in the second grating position $(8.5 \mathrm{~m})$.

5. Perform an arc and change to the third grating position $(1.5 \mathrm{~m})$.

1 See http://www.ing.iac.es/Astronomy/instruments/isis/ L3spectroscopy_v4.html 
Table 1. Multiple systems observed with lucky spectroscopy in this paper.

\begin{tabular}{|c|c|c|c|c|c|c|c|c|c|c|c|c|}
\hline Name & $\mathrm{PC}$ & $\mathrm{SC}$ & WDS ID & $B_{\mathrm{P}}$ & $\begin{array}{c}\text { Even. date } \\
\text { (YYMMDD) }\end{array}$ & Det. & $n_{\exp }$ & $n_{\text {used }}$ & $\begin{array}{l}t_{\text {exp. }} \\
(\mathrm{s})\end{array}$ & $\begin{array}{c}d \\
\left({ }^{\prime \prime}\right)\end{array}$ & $\begin{array}{l}\text { PA } \\
\left({ }^{\circ}\right)\end{array}$ & $\begin{array}{c}\Delta B \\
(\mathrm{mag})\end{array}$ \\
\hline \multirow[t]{2}{*}{ FN CMa } & \multirow[t]{2}{*}{ A } & \multirow[t]{2}{*}{ B } & \multirow[t]{2}{*}{ J07067-1118 } & \multirow[t]{2}{*}{5.8} & 190220 & $\mathrm{EEV}$ & 120 & 60 & 1.00 & 0.57 & 109 & 1.2 \\
\hline & & & & & 190917 & $\mathrm{EEV}$ & 100 & 15 & 1.00 & 0.57 & 109 & 1.2 \\
\hline $6 \mathrm{Cas}$ & A & $\mathrm{B}$ & $\mathrm{J} 23488+6213$ & 6.3 & 180729 & $\mathrm{EEV}$ & 143 & 65 & 1.00 & 1.50 & 194 & 2.3 \\
\hline \multirow[t]{2}{*}{$\alpha$ Sco } & \multirow[t]{2}{*}{ A } & \multirow[t]{2}{*}{$\mathrm{B}$} & \multirow[t]{2}{*}{ J16294-2626 } & \multirow[t]{2}{*}{2.8} & 120708 & EEV & 15 & 15 & 4.33 & 2.57 & 297 & 0.0 \\
\hline & & & & & 180730 & $\mathrm{EEV}$ & 100 & 23 & 1.00 & 2.70 & 273 & 3.6 \\
\hline$\sigma \mathrm{Sco}$ & $\mathrm{Aa}$ & $\mathrm{Ab}$ & J16212-2536 & 3.2 & 180730 & EEV & 100 & 13 & 1.00 & 0.47 & 242 & 2.1 \\
\hline CS Cam & A & $\mathrm{B}$ & J03291+5956 & 4.6 & 190917 & EEV & 100 & 66 & 1.00 & 2.30 & 159 & 4.2 \\
\hline \multirow{3}{*}{$\sigma$ Ori } & \multirow{3}{*}{$\mathrm{Aa}, \mathrm{Ab}$} & \multirow[t]{3}{*}{ B } & \multirow{3}{*}{ J05387-0236 } & \multirow[t]{3}{*}{4.4} & 170907 & $\mathrm{EEV}$ & 100 & 7 & 1.00 & 0.26 & 84 & 1.2 \\
\hline & & & & & 181203 & EEV & 100 & 12 & 1.00 & 0.25 & 67 & 1.2 \\
\hline & & & & & 190917 & EEV & 100 & 10 & 1.00 & 0.26 & 84 & 1.2 \\
\hline \multirow[t]{2}{*}{ HD 5005} & \multirow[t]{2}{*}{ A } & \multirow[t]{2}{*}{$\mathrm{B}$} & \multirow[t]{2}{*}{$\mathrm{J} 00528+5638$} & \multirow[t]{2}{*}{8.6} & 181219 & QUC & 1004 & $100-402$ & 1.00 & 1.53 & 82 & 1.6 \\
\hline & & & & & 190906 & EEV & 100 & 58 & 1.00 & 1.53 & 82 & 1.6 \\
\hline HD 51756 & A & B & J06585-0301 & 7.8 & 181219 & QUC & 1004 & $26-51$ & 1.00 & 0.63 & 100 & 0.3 \\
\hline HD 218195 & $\mathrm{~A}$ & B & $\mathrm{J} 23052+5815$ & 8.7 & 181220 & QUC & 502 & $216-365$ & 1.00 & 0.94 & 81 & 2.7 \\
\hline HD 219460 & A & B & $\mathrm{J} 23152+6027$ & 11.0 & 181220 & QUC & 502 & $423-499$ & 1.00 & 1.38 & 127 & 0.3 \\
\hline HD 8768 & A & B & $\mathrm{J} 01281+6317$ & 9.3 & 181220 & QUC & 502 & $138-247$ & 1.00 & 0.66 & 28 & 2.8 \\
\hline $\mathrm{BD}+60544$ & A & $\mathrm{B}$ & $\mathrm{J} 02411+6108$ & 10.4 & 181220 & QUC & 502 & $403-502$ & 1.00 & 1.91 & 170 & 0.8 \\
\hline HD 17520 & A & $\mathrm{B}$ & $\mathrm{J} 02512+6023$ & 10.3 & 181220 & QUC & 502 & $262-279$ & 1.00 & 0.32 & 298 & 0.7 \\
\hline HD 24431 & A & B & $\mathrm{J} 03556+5238$ & 7.2 & 181220 & QUC & 502 & $209-273$ & 1.00 & 0.72 & 177 & 2.9 \\
\hline HD 164492 & A & B & J18024-2302 & 7.6 & 190914 & EEV & 100 & 71 & 1.00 & 6.19 & 20 & 3.6 \\
\hline HD 168021 & A & B & J18187-1837 & 7.4 & 190421 & EEV & 100 & 33 & 1.00 & 0.48 & 137 & 1.0 \\
\hline HD 193443 & A & B & $\mathrm{J} 20189+3817$ & 8.3 & 190731 & $\mathrm{EEV}$ & 100 & 13 & 1.00 & 0.14 & 258 & 0.3 \\
\hline HD 194649 & A & B & $\mathrm{J} 20254+4014$ & 10.6 & 190914 & EEV & 100 & 95 & 1.00 & 0.30 & 214 & 0.7 \\
\hline HD 191201 & A & B & $\mathrm{J} 20074+3543$ & 7.8 & 190914 & $\mathrm{EEV}$ & 100 & 47 & 1.00 & 1.00 & 84 & 1.8 \\
\hline
\end{tabular}

Notes. PC and SC refer to the primary and secondary visual components, respectively, and $B_{\mathrm{P}}$ is the $B$-band magnitude of the primary. For each observation we give the evening date, the detector used (EEV for EEV12, QUC for QUCAM3), the number of total exposures, the number of exposures used for lucky spectroscopy (single value for EEV12, range for QUCAM3), the exposure time (for one exposure), the separation $d$ (measured from the data), the position angle used for the slit (which may be different from the one for the system), and the magnitude difference in $B$ (measured from the data).

6. Obtain 500 exposures of $1.0 \mathrm{~s}$ each in the third grating position $(8.5 \mathrm{~m})$.

7. Perform an arc and change to the first grating position $(1.5 \mathrm{~m})$.

This yields 34 min per object, a similar number as before, but with $500 \mathrm{~s}$ of total exposure time as opposed to $100 \mathrm{~s}$ (QUCAM3 has a slightly lower quantum efficiency than EEV12, but this effect is small). QUCAM3 might therefore be expected to be a more appropriate setup for faint stars, where the $\mathrm{S} / \mathrm{N}$ per frame is low. For bright stars, the advantage is lost because (a) the $\mathrm{S} / \mathrm{N}$ per frame is high and EEV12 has the advantages of being a simpler setup (fewer chances of making mistakes or having variable quality issues as a function of wavelength); (b) it covers a wider wavelength range; (c) is easier to schedule because it is the default detector for the instrument; and (d) for the brightest objects, such as some of the ones in LS I, an exposure time of $0.1 \mathrm{~s}$ for is possible to avoid saturation (something which is not allowed in QUCAM3 because the minimum exposure time is set by the readout time of the image). We test the hypothesis about faint stars below with one example.

\subsection{Complementary data}

For some of our systems we presented lucky imaging results obtained with AstraLux at the $2.2 \mathrm{~m}$ CAHA telescope in Maíz Apellániz (2010) and MONOS I. Here we present additional separations, position angles, and magnitude differences in Table 3. The references above describe the details of the setup and reduction, which have not changed since the publication of MONOS I. In this paper we also refer to the information listed in the Washington Double Star (WDS) catalog (Mason et al. 2001) and use astrometric results from Gaia DR2.

In addition to the new lucky spectroscopy data, we also include in this paper results from regular long-slit or IFU spectroscopy from GOSSS. The purpose is to compare the two methods and to study an additional component in one of the systems. For another system, we also use STIS/HST data that have been presented in STIS I as a comparison. Finally, we also use groundbased high-resolution spectroscopy from LiLiMaRlin for some systems.

\section{Results}

We present our results in Table 2 . We discuss each system individually below, comparing the new information with the previously available data. As with our previous results, the spectral classifications and the spectra themselves will be made made available at the GOSC web site ${ }^{2}$.

\subsection{FN CMa A,B, a bright O-type system}

This fifth-magnitude system, also known as HD 53974 A,B, was classified as B0.5 IV by Morgan et al. (1955), and Simbad lists different classifications ranging in spectral subtypes from

2 http://gosc.cab.inta-csic.es 
Table 2. Spectral classifications.

\begin{tabular}{|c|c|c|c|c|c|c|c|c|}
\hline Name & GOSSS ID & RA (J2000) & Dec (J2000) & ST & $\mathrm{LC}$ & Qual. & Second. & Notes \\
\hline FN CMa A & GBS 224.71-01.79_01 & 07:06:40.767 & $-11: 17: 38.46$ & B 0.7 & $\mathrm{Ib}$ & & $\ldots$ & SB1 in LiLiMaRlin \\
\hline FN CMa B & GOS 224.71-01.79_02 & 07:06:40.803 & $-11: 17: 38.71$ & O6 & $\mathrm{V}$ & $((f)) \mathrm{z}$ & $\ldots$ & $\ldots$ \\
\hline 6 Cas A & GAS $115.71+00.22 \_02$ & 23:48:50.166 & $+62: 12: 52.22$ & A3 & Ia & $\ldots$ & $\ldots$ & $\ldots$ \\
\hline 6 Cas B & GOS 115.71+00.22_01 & 23:48:50.108 & $+62: 12: 50.75$ & 09.5 & II & $\ldots$ & $\ldots$ & $\ldots$ \\
\hline$\alpha \operatorname{Sco} \mathrm{A}$ & GLS 351.95+15.06_01 & $16: 29: 24.461$ & $-26: 25: 55.21$ & M1.5 & $\mathrm{Iab}$ & $\ldots$ & $\ldots$ & $\ldots$ \\
\hline$\alpha$ Sco B & GBS 351.95+15.06_02 & $16: 29: 24.275$ & $-26: 25: 55.04$ & B2 & $\mathrm{V}$ & $\mathrm{n}$ & $\ldots$ & 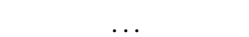 \\
\hline$\sigma \mathrm{Sco} \mathrm{Aa}$ & GBS $351.31+17.00 \_01$ & $16: 21: 11.313$ & $-25: 35: 34.09$ & B1 & III & $\ldots$ & $\ldots$ & SB2 in LiLiMaRlin \\
\hline$\sigma \mathrm{Sco} \mathrm{Ab}$ & GBS $351.31+17.00 \_02$ & $16: 21: 11.282$ & $-25: 35: 34.30$ & B1: & $\mathrm{V}$ & $\ldots$ & $\ldots$ & $\ldots$ \\
\hline CS Cam A & GBS 141.50+02.88_01 & 03:29:04.136 & $+59: 56: 25.20$ & B9 & Ia & $\ldots$ & $\ldots$ & $\ldots$ \\
\hline CS Cam B & GBS 141.50+02.88_02 & 03:29:04.252 & $+59: 56: 22.88$ & B2 & III & $\ldots$ & $\ldots$ & $\ldots$ \\
\hline$\sigma$ Ori $\mathrm{Aa}, \mathrm{Ab}$ & GOS 206.82-17.34_01 & 05:38:44.765 & $-02: 36: 00.25$ & 09.5 & $\mathrm{~V}$ & $\ldots$ & B $0.2 \mathrm{~V}$ & In LS I + MONOS I \\
\hline$\sigma$ Ori B & GBS 206.82-17.34_02 & $05: 38: 44.782$ & $-02: 36: 00.27$ & B0.2 & V & (n) & $\ldots$ & In LS I + MONOS I \\
\hline HD $5005 \mathrm{~A}$ & GOS 123.12-06.24_01 & 00:52:49.206 & $+56: 37: 39.49$ & $\mathrm{O} 4.5$ & $\mathrm{~V}$ & $((\mathrm{fc})) \mathrm{z}$ & $\ldots$ & In GOSSS I \\
\hline HD 5005 B & GBS $123.12-06.24 \_03$ & 00:52:49.390 & $+56: 37: 39.71$ & B0 & $\mathrm{V}$ & $\ldots$ & $\ldots$ & In GOSSS I \\
\hline HD 51756 A & GBS $216.42+00.18 \_01$ & $06: 58: 28.180$ & $-03: 01: 25.44$ & B0 & IV & $\ldots$ & $\ldots$ & SB1? in LiLiMaRlin \\
\hline HD 51756 B & GOS 216.42+00.18_02 & $06: 58: 28.221$ & $-03: 01: 25.57$ & O9.7 & IV & (n) & $\ldots$ & \\
\hline HD 218195 A & GOS 109.32-01.79_01 & 23:05:12.928 & $+58: 14: 29.34$ & 08.5 & III & Nstr & $\ldots$ & In GOSSS I + II \\
\hline HD 218195 B & GBS 109.32-01.79_02 & 23:05:13.043 & $+58: 14: 29.49$ & B1.5 & $\mathrm{V}$ & $\ldots$ & $\ldots$ & SB2? in LiLiMaRlin \\
\hline HD $219460 \mathrm{~A}$ & GBS 111.33-00.24_01 & $23: 15: 12.394$ & $+60: 27: 01.84$ & B 0.7 & II & $\ldots$ & B1: III: & $\ldots$ \\
\hline WR 157 & GWS 111.33-00.24_02 & $23: 15: 12.542$ & $+60: 27: 01.00$ & WN5 & $\ldots$ & $\ldots$ & $\ldots$ & $\ldots$ \\
\hline HD $8768 \mathrm{~A}$ & GOS 127.03+00.70_01 & 01:28:03.153 & $+63: 16: 57.20$ & OC9.2 & II & $\ldots$ & $\ldots$ & $\ldots$ \\
\hline HD 8768 B & GBS $127.03+00.70 \_02$ & 01:28:03.198 & $+63: 16: 57.78$ & B0.7: & $\mathrm{V}$ & $\ldots$ & $\ldots$ & $\ldots$ \\
\hline $\mathrm{BD}+60544 \mathrm{~A}$ & GOS 135.78+01.03_01 & 02:41:08.194 & $+61: 08: 07.02$ & 09.5 & IV & $\ldots$ & $\ldots$ & $\ldots$ \\
\hline $\mathrm{BD}+60544 \mathrm{~B}$ & GBS 135.78+01.03_02 & 02:41:08.244 & $+61: 08: 05.14$ & B1 & $\mathrm{V}$ & $\ldots$ & $\ldots$ & $\ldots$ \\
\hline HD $17520 \mathrm{~A}$ & GOS 137.22+00.88_01 & 02:51:14.434 & $+60: 23: 09.97$ & 09.2 & V & $\mathrm{e}$ & $\ldots$ & $\begin{array}{c}\text { In GOSSS III } \\
\text { SB1 in LiLiMaRlin }\end{array}$ \\
\hline HD $17520 \mathrm{~B}$ & GOS 137.22+00.88_02 & 02:51:14.396 & $+60: 23: 10.12$ & $\mathrm{O} 8$ & $\mathrm{~V}$ & $\mathrm{z}$ & $\ldots$ & In GOSSS III \\
\hline HD $24431 \mathrm{~A}$ & GOS 148.84-00.71_01 & 03:55:38.420 & $+52: 38: 28.75$ & O9 & III & $\ldots$ & $\ldots$ & $\begin{array}{c}\text { In GOSSS I } \\
\text { SB1 in LiLiMaRlin }\end{array}$ \\
\hline HD $24431 \mathrm{~B}$ & GBS 148.84-00.71_02 & 03:55:38.424 & $+52: 38: 28.03$ & B1.5 & $\mathrm{V}$ & $\ldots$ & $\ldots$ & $\ldots$ \\
\hline HD 164492 A & GOS 007.00-00.25_01 & $18: 02: 23.553$ & $-23: 01: 51.06$ & 07.5 & $\mathrm{~V}$ & $\mathrm{z}$ & $\ldots$ & In GOSSS II \\
\hline HD 164492 B & GBS 007.00-00.25_03 & $18: 02: 23.701$ & $-23: 01: 45.14$ & $\mathrm{~B} 2$ & V & $\mathrm{nn}$ & $\ldots$ & $\ldots$ \\
\hline HD $164492 \mathrm{C}$ & GBS 007.00-00.25_02 & 18:02:23.132 & $-23: 02: 00.17$ & B1 & V & $\ldots$ & $\ldots$ & SB3 in LiLiMaRlin \\
\hline HD $168021 \mathrm{~A}$ & GBS 012.70-01.47_01 & $18: 18: 43.260$ & $-18: 37: 10.81$ & B0 & Ia & $\ldots$ & $\ldots$ & $\ldots$ \\
\hline HD 168021 B & GBS 012.70-01.47_02 & $18: 18: 43.281$ & $-18: 37: 11.18$ & B 0.2 & II & $\ldots$ & $\ldots$ & $\ldots$ \\
\hline HD $168021 \mathrm{C}$ & GBS 012.71-01.47_01 & $18: 18: 44.204$ & $-18: 36: 59.94$ & B 0.5 & II & $\ldots$ & $\ldots$ & $\ldots$ \\
\hline \multirow[t]{2}{*}{ HD 193443 A } & GOS 076.15+01.28_01 & 20:18:51.707 & $+38: 16: 46.50$ & O8.5 & III: & $((\mathrm{f}))$ & $\ldots$ & In MONOS I \\
\hline & & & & O8.5 & III & ((f)) & $\ldots$ & Sp. cl. from STIS I \\
\hline \multirow[t]{2}{*}{ HD 193443 B } & GOS 076.15+01.28_02 & $20: 18: 51.697$ & $+38: 16: 46.48$ & O9.2: & IV: & $\ldots$ & $\ldots$ & In MONOS I \\
\hline & & & & 09.2 & IV & $\ldots$ & $\ldots$ & Sp. cl. from STIS I \\
\hline HD 194649 A & GOS 078.46+01.35_01 & $20: 25: 22.124$ & $+40: 13: 01.07$ & O5.5 & $\mathrm{V}$ & $((\mathrm{f}))$ & $09.5 \mathrm{~V}$ & In MONOS I \\
\hline HD 194649 B & GOS 078.46+01.35_02 & 20:25:22.109 & $+40: 13: 00.82$ & $\mathrm{O} 7$ & $\mathrm{~V}$ & $\mathrm{z}$ & $\ldots$ & In MONOS I \\
\hline HD $191201 \mathrm{~A}$ & GOS 072.75+01.78_01 & 20:07:23.684 & $+35: 43: 05.91$ & 09.5 & III & $\ldots$ & $\mathrm{B} 0 \mathrm{~V}$ & In MONOS I \\
\hline HD 191201 B & GOS 072.75+01.78_02 & $20: 07: 23.766$ & $+35: 43: 06.01$ & O9.7 & $\mathrm{V}$ & $\ldots$ & $\ldots$ & In MONOS I \\
\hline
\end{tabular}

Notes. GOS/GBS/GAS/GWS/GLS stand for Galactic O/B/A/WR/Late Star, respectively. The information in this table is also available in electronic form at the GOSC web site (http://gosc.cab.inta-csic.es).

B0 to B3. It is a visual binary with $d=0$ ".6 and $\Delta m=1.21 \mathrm{mag}$ listed in the WDS catalog. Rivinius et al. (2011) used spectral disentangling to obtain that the A visual component is a B0 III star and the B component is an O6 $\mathrm{V}((\mathrm{f}))$ background object because they deemed the magnitude difference to be too large to place two such stars at the same distance. FN CMa appears to be a single source with no parallax in Gaia DR2. To estimate its distance, we assumed that it is the same as component $\mathrm{C}$ in the system. FN CMa C (Gaia DR2 3046209991397371392) has a parallax of $0.6844 \pm 0.0509$ mas and a renormalised unit weight error (RUWE) of 0.98. For its distance, we used the Bayesian prior described by Maíz Apellániz (2001, 2005) with the updated Galactic (young) disk parameters from Maíz Apellániz et al. (2008), applied a parallax zeropoint of $0.040 \pm 0.010$ mas (Maíz Apellániz et al. 2020b), and obtained $1.48_{-0.11}^{+0.12} \mathrm{kpc}$.
We attempted to separate the two components of the system on three occasions and were successful in two of them (Fig. 1). The primary is indeed an early-B star, but of later spectral subtype (B0.7) and higher luminosity class (Ib) than claimed by Rivinius et al. (2011). The secondary is indeed an O6 dwarf, but with z suffix, thus likely underluminous for its spectral classification (Arias et al. 2016). These differences with the Rivinius et al. (2011) classifications explain the $\Delta m$ between the two components and allow them to be at the same distance, a more likely arrangement than a chance alignment between two such bright stars. The two components are cleanly separated, with no sign of He II $\lambda 4542$ in the primary or of Si III or O II lines in the secondary, and with radically different (expected for their spectral types) behaviors in the $4630-4650 \AA$ region. No appreciable differences are seen between the two epochs (but see below for the high spectral resolution results). The combined spectrum 
Table 3. Measurements for visual pairs using our AstraLux lucky images.

\begin{tabular}{|c|c|c|c|c|c|c|c|c|c|c|}
\hline Pair & $\begin{array}{c}\text { Even. date } \\
\text { (YYMMDD) }\end{array}$ & $\begin{array}{c}\text { HJD-2 } 400000 \\
\text { (d) }\end{array}$ & $\begin{array}{c}d \\
\left({ }^{\prime \prime}\right)\end{array}$ & $\begin{array}{c}\theta \\
(\mathrm{deg})\end{array}$ & $\begin{array}{c}\Delta V \\
(\mathrm{mag})\end{array}$ & $\begin{array}{c}\Delta \mathrm{H} \alpha \\
(\mathrm{mag})\end{array}$ & $\begin{array}{c}\Delta i \\
(\mathrm{mag})\end{array}$ & $\begin{array}{c}\Delta z \\
(\mathrm{mag})\end{array}$ & $\begin{array}{c}\Delta z n \\
(\mathrm{mag})\end{array}$ & $\begin{array}{c}\Delta Y \\
(\mathrm{mag})\end{array}$ \\
\hline \multirow[t]{2}{*}{ FN CMa A,B } & 121002 & 56203.70 & $0.577 \pm 0.005$ & $115.58 \pm 0.11$ & $\ldots$ & $\ldots$ & $\ldots$ & $\ldots$ & $1.66 \pm 0.02$ & $\ldots$ \\
\hline & 181126 & 58449.63 & $0.581 \pm 0.005$ & $115.39 \pm 0.11$ & $\ldots$ & $\ldots$ & $\ldots$ & $\ldots$ & $1.63 \pm 0.02$ & $\ldots$ \\
\hline \multirow[t]{2}{*}{6 Cas A,B } & 121002 & 56203.52 & $1.497 \pm 0.003$ & $195.69 \pm 0.04$ & $2.45 \pm 0.01$ & $\ldots$ & $\ldots$ & $3.05 \pm 0.01$ & $\ldots$ & $\ldots$ \\
\hline & 130918 & 56554.63 & $1.496 \pm 0.003$ & $195.81 \pm 0.06$ & $\ldots$ & $\ldots$ & $2.78 \pm 0.02$ & $\ldots$ & $\ldots$ & $\ldots$ \\
\hline$\alpha \mathrm{Sco} A, \mathrm{~B}$ & 190615 & 58650.44 & $2.743 \pm 0.021$ & $276.96 \pm 0.23$ & $\ldots$ & $6.09 \pm 0.07$ & $\ldots$ & $\ldots$ & $7.45 \pm 0.11$ & $8.01 \pm 0.10$ \\
\hline$\sigma \mathrm{Sco} \mathrm{Aa}, \mathrm{Ab}$ & 190616 & 58651.40 & $0.423 \pm 0.003$ & $217.17 \pm 0.22$ & $\ldots$ & $\ldots$ & $\ldots$ & $\ldots$ & $2.10 \pm 0.09$ & $2.25 \pm 0.07$ \\
\hline \multirow[t]{3}{*}{ HD 5005 A,B } & 071112 & 54417.44 & $1.526 \pm 0.002$ & $82.12 \pm 0.04$ & $\ldots$ & $\ldots$ & $\ldots$ & $1.59 \pm 0.01$ & $\ldots$ & $\ldots$ \\
\hline & 130918 & 56554.64 & $1.526 \pm 0.002$ & $82.25 \pm 0.04$ & $\ldots$ & $\ldots$ & $1.60 \pm 0.01$ & $\ldots$ & $\ldots$ & $\ldots$ \\
\hline & 181127 & 58450.36 & $1.526 \pm 0.002$ & $82.19 \pm 0.04$ & $\ldots$ & $\ldots$ & $1.57 \pm 0.01$ & $1.59 \pm 0.01$ & $\ldots$ & $\ldots$ \\
\hline \multirow[t]{2}{*}{ HD 51756 A,B } & 121013 & 56204.69 & $0.705 \pm 0.001$ & $102.94 \pm 0.04$ & $\ldots$ & $\ldots$ & $\ldots$ & $0.29 \pm 0.01$ & $\ldots$ & $\ldots$ \\
\hline & 130919 & 56555.69 & $0.706 \pm 0.001$ & $102.83 \pm 0.04$ & $\ldots$ & $\ldots$ & $0.32 \pm 0.01$ & $\ldots$ & $\ldots$ & $\ldots$ \\
\hline \multirow[t]{2}{*}{ HD 218195 A,B } & 110913 & 55818.48 & $0.944 \pm 0.001$ & $80.01 \pm 0.07$ & $\ldots$ & $\ldots$ & $\ldots$ & $2.66 \pm 0.04$ & $\ldots$ & $\ldots$ \\
\hline & 130918 & 56554.52 & $0.943 \pm 0.001$ & $80.01 \pm 0.08$ & $\ldots$ & $\ldots$ & $2.69 \pm 0.03$ & $\ldots$ & $\ldots$ & $\ldots$ \\
\hline HD 219460 A,B & 110913 & 55818.45 & $1.371 \pm 0.001$ & $128.00 \pm 0.04$ & $\ldots$ & $\ldots$ & $\ldots$ & $-0.12 \pm 0.01$ & $\ldots$ & $\ldots$ \\
\hline \multirow[t]{3}{*}{ HD 8768 A,B } & 081021 & 54761.63 & $0.662 \pm 0.002$ & $27.97 \pm 0.34$ & $\ldots$ & $\ldots$ & $\ldots$ & $2.62 \pm 0.09$ & $\ldots$ & $\ldots$ \\
\hline & 110913 & 55818.50 & $0.658 \pm 0.002$ & $28.42 \pm 0.18$ & $\ldots$ & $\ldots$ & $\ldots$ & $2.73 \pm 0.04$ & $\ldots$ & $\ldots$ \\
\hline & 130918 & 56554.65 & $0.657 \pm 0.004$ & $28.57 \pm 0.15$ & $\ldots$ & $\ldots$ & $2.86 \pm 0.14$ & $\ldots$ & $\ldots$ & $\ldots$ \\
\hline $\mathrm{BD}+60544 \mathrm{~A}, \mathrm{~B}$ & 180918 & 58380.59 & $1.948 \pm 0.001$ & $168.51 \pm 0.04$ & $\ldots$ & $\ldots$ & $\ldots$ & $0.76 \pm 0.02$ & $\ldots$ & $\ldots$ \\
\hline \multirow[t]{2}{*}{ HD 17520 A,B } & 080118 & 54484.38 & $0.317 \pm 0.001$ & $298.72 \pm 0.07$ & $\ldots$ & $\ldots$ & $\ldots$ & $0.69 \pm 0.01$ & $\ldots$ & $\ldots$ \\
\hline & 130918 & 56554.66 & $0.320 \pm 0.001$ & $299.05 \pm 0.08$ & $\ldots$ & $\ldots$ & $0.71 \pm 0.02$ & $\ldots$ & $\ldots$ & $\ldots$ \\
\hline \multirow[t]{2}{*}{ HD 24431 A,B } & 080118 & 54484.41 & $0.734 \pm 0.001$ & $177.18 \pm 0.15$ & $\ldots$ & $\ldots$ & $\ldots$ & $2.83 \pm 0.09$ & $\ldots$ & $\ldots$ \\
\hline & 110913 & 55818.59 & $0.737 \pm 0.004$ & $176.65 \pm 0.11$ & $\ldots$ & $\ldots$ & $\ldots$ & $3.10 \pm 0.17$ & $\ldots$ & $\ldots$ \\
\hline HD 164492 A,B & 130919 & 56555.30 & $6.261 \pm 0.001$ & $20.37 \pm 0.04$ & $\ldots$ & $\ldots$ & $\ldots$ & $3.01 \pm 0.05$ & $\ldots$ & $\ldots$ \\
\hline HD 164492 A,C & 130919 & 56555.30 & $10.707 \pm 0.008$ & $211.93 \pm 0.04$ & $\ldots$ & $\ldots$ & $\ldots$ & $1.35 \pm 0.05$ & $\ldots$ & $\ldots$ \\
\hline HD 164492 A,H & 130919 & 56555.30 & $1.489 \pm 0.019$ & $345.03 \pm 0.10$ & $\ldots$ & $\ldots$ & $\ldots$ & $5.21 \pm 0.19$ & $\ldots$ & $\ldots$ \\
\hline
\end{tabular}

Notes. The evening date, Heliocentric Julian Date (HJD), separation $(d)$, position angle $(\theta)$, and magnitude difference is given in each case. Six different filters were used: Johnson $V, \mathrm{H} \alpha$, SDSS $i$ and $z, z n$ (a narrow filter with a central wavelength similar to that of $z$ ), and $Y$, in order of increasing central wavelength.

(top in Fig. 1) can be classified as B0.2 III, but some peculiarities are seen in it that indicate a composite nature, such as the inability to consistently fit the Balmer profiles and the Si lines at the same time. This is a good example of the advantage of lucky spectroscopy (spatial disentangling) over spectral disentangling: the latter can introduce artifacts that lead to erroneous spectral characterizations. In this particular case, we suspect that in the Rivinius et al. (2011) disentangling some He II $\lambda 4542$ may have remained in the spectrum of the primary (but as they did not publish the disentangled spectra, we cannot be certain).

We also observed this system with lucky imaging (Table 3 ). The separations and position angles are compatible with the WDS data, but the magnitude difference is just barely so: HIPPARCOS gives $\Delta H=1.357 \mathrm{mag}$, and we only expect a color term of $\sim 0.1 \mathrm{mag}$ with $z$ photometry. There is no appreciable motion in the six-year span between the two epochs, and there is no obvious trend when we consider the positions in the WDS catalog that stretch to more than 100 years ago.

We have nine epochs of this system in the high-resolution (A+B combined) LiLiMaRlin spectra. The He II lines (which originate mostly in the $\mathrm{O}$ star) do not show profile changes or velocity shifts. The Si III lines (which originate mostly in the B supergiant) show velocity shifts, and the He I lines (which originate in both but mostly in the B supergiant) show profile variations and/or velocity shifts. This is consistent with the finding by Rivinius et al. (2011) that the B-type star is an SB1 with a $117.55 \pm 0.33 \mathrm{~d}$ period, making FN CMa a quadruple system (including the $\mathrm{C}$ component that lies $18^{\prime \prime}$ away).

\subsection{Cas $A, B$, the only known $O$ star with an $A$ supergiant companion}

This fifth-magnitude system, also known as HD 223385 A,B, was classified as A3 Ia + sec by Morgan et al. (1953), where we explicitly add "sec" to indicate that the plus sign does not refer to a hypergiant classification (a common source of confusion, note that hypergiants had not been introduced at the time). The B companion is currently listed in the WDS cata$\log$ with $d=11^{\prime \prime} 5, \mathrm{PA}=194^{\circ}$, and $\Delta m=2.29$ mag measured in 2015. Talavera \& Gómez de Castro (1987) were the first to suggest that the A supergiant had an O-type companion based on the composite UV spectrum, but they favoured a spectroscopic, not a visual binary. The two components have separate entries in Gaia DR2, but their parallaxes cannot be used to derive precise distances: the parallax of A is negative and that of B has a RUWE of 5.15, indicating poor astrometric quality, likely caused by the proximity of the A component. The Gaia DR2 $\Delta G$ should be reliable, with a value of $2.529 \pm 0.016 \mathrm{mag}$ for the A.B pair, but the $\Delta G_{\mathrm{BP}}$ and $\Delta G_{\mathrm{RP}}$ are likely not because they are measured by aperture photometry and the two components are too close. The Gaia DR2 positions yield a separation of 1 ".5016 and a position angle of $195.89^{\circ}$.

Because we cannot use the Gaia DR2 data for 6 Cas A,B to obtain a reliable distance to the system, we searched for nearby stars that may be associated with it. The WDS cata$\log$ lists a C component, but it has a RUWE of 3.87, therefore we cannot use it. Instead, we used a variation of the method used in Maíz Apellániz (2019). We started with the HIPPARCos 


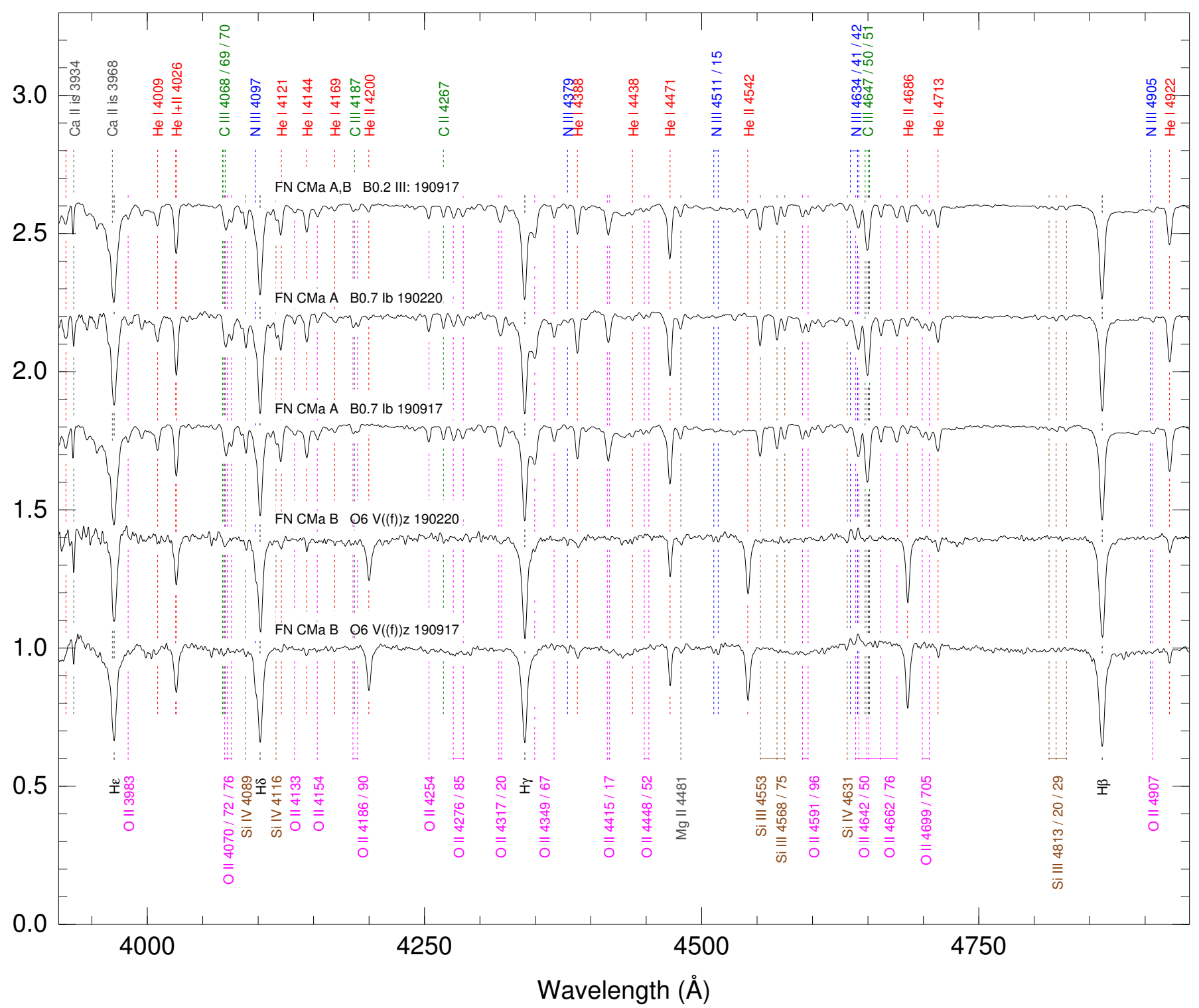

Fig. 1. Rectified spectrograms for FN CMa at the GOSSS spectral resolution of $R \sim 2500$ and on the stellar reference frame. For each spectrogram, the name, spectral type, and evening date (YYMMDD) are shown. The top spectrogram is the weighted combination of the two components for one of the epochs. Main atomic and ISM lines are indicated.

Table 4. Gaia DR2 astrometric data for four stars in the vicinity of 6 Cas with similar parallaxes and proper motions, and their aggregate results.

\begin{tabular}{lccc}
\hline \hline Gaia DR2 ID & $\begin{array}{c}\varpi \\
\text { (mas) }\end{array}$ & $\begin{array}{c}\mu_{\alpha *} \\
(\mathrm{mas} / \mathrm{a})\end{array}$ & $\begin{array}{c}\mu_{\delta} \\
(\mathrm{mas} / \mathrm{a})\end{array}$ \\
\hline 2012942869740260096 & $0.3233 \pm 0.0319$ & $-3.600 \pm 0.046$ & $-1.781 \pm 0.043$ \\
2012942805332107136 & $0.3457 \pm 0.0189$ & $-3.417 \pm 0.030$ & $-1.421 \pm 0.028$ \\
2012942496094500096 & $0.3272 \pm 0.0257$ & $-3.442 \pm 0.039$ & $-1.387 \pm 0.035$ \\
2012942564813963264 & $0.3628 \pm 0.0263$ & $-3.569 \pm 0.041$ & $-1.479 \pm 0.038$ \\
\hline & $0.3428 \pm 0.0402$ & $-3.482 \pm 0.063$ & $-1.465 \pm 0.063$ \\
\hline
\end{tabular}

Notes. The aggregate results use external uncertainties and include the spatial covariance terms of Lindegren et al. (2018).

proper motion for 6 Cas, and we searched for Gaia DR2 stars in the vicinity with (a) similar proper motions, (b) good RUWE, (c) $G$ magnitudes brighter than 15 , (d) similar blue colors, and (e) self-compatible parallaxes. We found four such stars and list them in Table 4. We combined their parallaxes using the technique described in Campillay et al. (2019), including the use of external uncertainties and a spatial covariance term (Lindegren et al. 2018), and applied the same parallax zeropoint and prior as before. The resulting distance to this group of stars is $2.78_{-0.29}^{+0.37} \mathrm{kpc}$, which we use here for 6 Cas itself.

We successfully separated the two components of this system on eight occasions with lucky spectroscopy. No significant variations in the spectral types were observed between epochs, therefore we only plot in Fig. 2 one lucky spectroscopy epoch (including the combined spectrum) along with another epoch of the system for which we were able to obtain separate spectra with FRODOspec/LT (regular GOSSS spectroscopy). The two spectra are cleanly separated despite their very different spectral types, with no sign of cross-contamination. 6 Cas B has an O9.5 II spectral type with no sign of any anomaly. The effect of the $\mathrm{B}$ component on the combined spectrum is very weak and is only detected in a few lines such as Si IV $\lambda 4089$, C III $\lambda \lambda \lambda 4647,4650,4651$, He II $\lambda 4686$, and He I $\lambda 4713$, which are located in wavelength regions without strong lines from the 


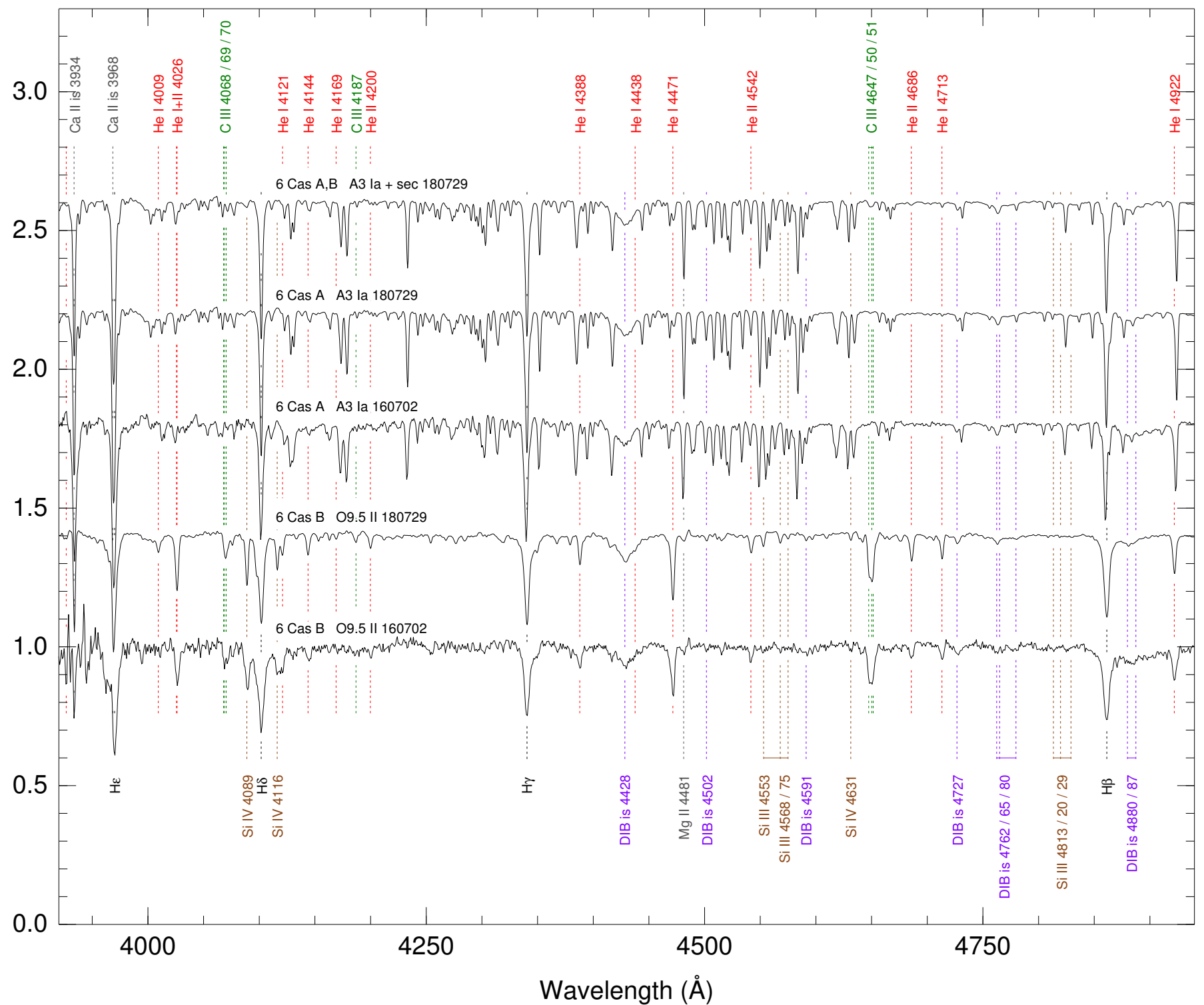

Fig. 2. Rectified spectrograms for 6 Cas at the GOSSS spectral resolution of $R \sim 2500$ and on the stellar reference frame. For each spectrogram, the name, spectral type, and evening date (YYMMDD) are shown. The 160702 data were obtained with FRODOspec/LT, i.e., not with lucky spectroscopy. The top spectrogram is the weighted combination of the two components for the 180729 epoch. Main atomic and ISM lines are indicated.

A supergiant. The FRODOspec spectrograms indicate that this system can also be resolved with regular spectroscopy, but at a cost. While the two spectra for the A component in Fig. 2 are nearly identical in spectral resolution and $\mathrm{S} / \mathrm{N}$, the lucky spectrogram for the $\mathrm{B}$ component has a higher $\mathrm{S} / \mathrm{N}$ than its regular counterpart due to the reduced noise introduced in the spatial disentangling process.

We have two lucky-imaging epochs of this system (Table 3) spaced by a year and obtained prior to the average Gaia DR2 reference epoch (2015.5). When these points are combined with the historical data in the WDS catalog, there is little indication of a secular motion, except perhaps in the inward direction; most observations from the nineteenth century and from the beginning of the twentieth have separations of $11^{\prime \prime} 6-1{ }^{\prime \prime} 7$. The observed magnitude differences in our lucky images are consistent with the $\Delta G$ value above and with the spectral types.

We have 12 LiLiMaRlin epochs covering from 2001 to 2020. The lines of the A supergiant show radial velocity variations with a peak-to-peak amplitude of $\sim 10 \mathrm{~km} \mathrm{~s}^{-1}$. The motion is not secular, but shows variations on timescales of days; pulsations are therefore the likely reason. The phenomenon has previously been reported by Aydin (1979). The signature of the B component is difficult to detect in the LiLiMaRlin spectra because of the additional dilution caused by the 1".5 separation and the circular apertures of less than $3^{\prime \prime}$ diameter used by échelle spectrographs. In some of our epochs, however, He II $\lambda 5412$ is clearly visible.

\section{3. $\alpha$ Sco $A, B$, the nearest $R S G+B$ system}

More than 100 red supergiant stars with B-type companions are known in the Galaxy (Pantaleoni González et al. 2020), of which $\alpha$ Sco (Antares) is the closest, with a HIPPARCos distance ( $\alpha$ Sco is not present in Gaia DR2) of $187_{-30}^{+44}$ pc (Maíz Apellániz et al. 2008). The companion was classified as B3 one century ago (Adams \& Joy 1921), and subsequent authors classified it as 

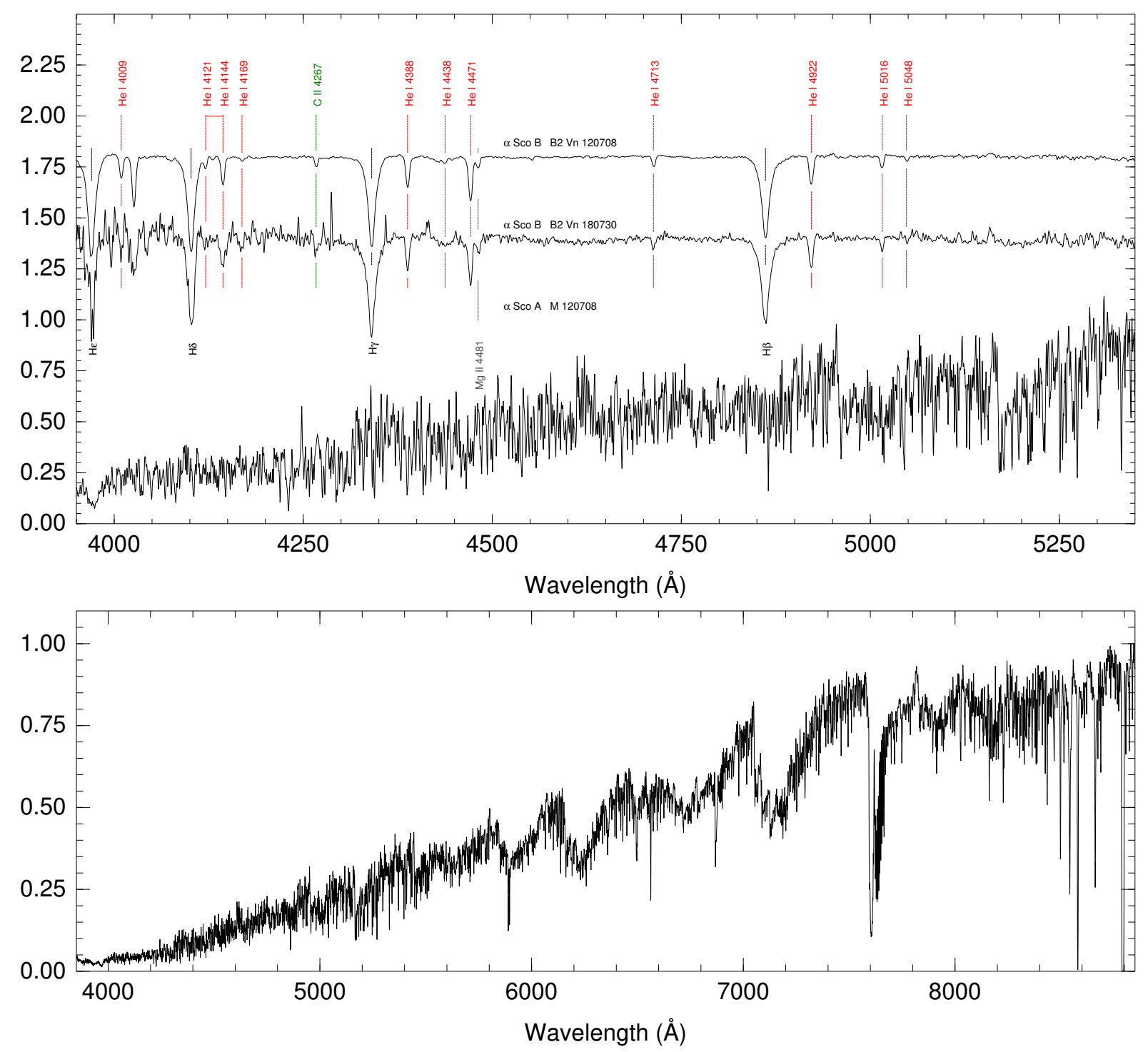

Fig. 3. Upper panel: lucky spectrograms for $\alpha$ Sco at the GOSSS spectral resolution of $R \sim 2500$ and on the stellar reference frame. For each spectrogram, the name, spectral type, and evening date (YYMMDD) are shown. The 120730 data were obtained with a position angle offset of $24^{\circ}$ from the A,B line, and the 180730 data were obtained with the slit aligned with the A,B line. The spectra of the B component have been rectified, while the spectrum of the A component has been dereddened and relative-flux calibrated. Main atomic lines are indicated. Lower panel: $\alpha$ Sco A spectrogram from LiLiMaRlin degraded to $R \sim 4000$, relative-flux calibrated, and not corrected for telluric absorption.

B4 V (Stone \& Struve 1954), B2.5 V (Garrison 1967), and B3 V (Corbally 1984). The differences in classification likely do not reflect real spectral variations, but are rather a consequence of the difficulty that exists in eliminating the light from the much brighter primary less than $3^{\prime \prime}$ away. The B star has not received much attention in the past decades other than the study of its associated emission nebula, which is rich in [Fe II] lines (Swings \& Preston 1978; Reimers et al. 2008). The magnitude difference between the two components is highly dependent on the band used (see below), as is expected for a pair composed of a red and a blue star (this pair is a favourite object among amateur astronomers who wish to separate visual binaries of extreme colors and small separations, the first author still remembers his excitement when he was able to separate Antares through an eyepiece for the first time 34 years ago).

We observed $\alpha$ Sco A,B with lucky spectroscopy on four occasions and we were successful in all of them, but with very different values of the $\mathrm{S} / \mathrm{N}$ for the $\mathrm{B}$ component. This effect is likely caused by the large magnitude difference. We measure this value in our data to be $\sim 3.6 \mathrm{mag}$ in the $B$ band, but with a significant difference between $4000 \AA$ and $5000 \AA$. The epoch with the best seeing (and consequently higher $\mathrm{S} / \mathrm{N}$ ) of the four epochs is the first epoch from 2012, when we first attempted lucky spectroscopy with ISIS/WHT. On that occasion we centered the slit on the B component and used a position angle offset of $24^{\circ}$ with respect to the A-B line. This reduced the effective separation slightly, but was heavily compensated for by the effect of making the flux from the two components similar in the $B$ band, which is the most important constraint in this case (see Table 1). We classify the B component as B2 Vn, which differs from previous results in two ways: the spectral subtype is earlier and the star shows a significant line broadening that is likely caused by rotation. We used the semiautomatized tool iacob-broad to measure the projected rotational velocity $(v \sin i)$ from a combined Fourier transform plus goodness-of-fit technique (see Simón-Díaz \& Herrero 2014 and references therein) 
using the top spectrum in Fig 3. The absence of strong metallic lines caused by the significant line broadening made us rely on He I lines. This means that the Stark broadening effect needs to be considered, and therefore, the amount of macroturbulent broadening cannot be adjusted. From the fitting of four different

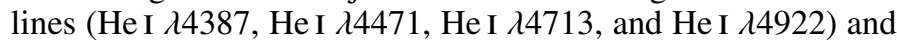
the agreement between results obtained by the two techniques we used as a consistency check (see, e.g., Berlanas et al. 2020), we obtain a $v \sin i$ for the B component of $260 \pm 30 \mathrm{~km} \mathrm{~s}^{-1}$. In this way, $\alpha$ Sco B is another example of a massive-star companion with high rotation (see LS I and STIS I).

Morgan \& Keenan (1973) selected $\alpha$ Sco A as the MK M1.5 Iab standard, although Keenan \& McNeil (1989) later gave it a slightly lower luminosity at M1.5 Iab-Ib. Many spectral classifications in the literature have indicated earlier types, but the strength of the $\mathrm{TiO}$ band system in the spectrum rules them out We used the Mercator spectrogram from LiLiMaRlin shown in Fig. 3 to classify $\alpha$ Sco A following the procedures described in Dorda et al. (2018) and their set of standards at $R=4000$. Direct comparison to HD 206936 (M2 Ia) and HD 36389 (M2 IabIb) shows that all the temperature-sensitive $\mathrm{TiO}$ bandheads are essentially identical in $\alpha$ Sco A, although the bands of $\alpha$ Ori, nominally M1-2 Ia-Iab, are very slightly stronger. The strength of the Ca II triplet and other luminosity indicators in its range agree well with those in the blue, and we consider the M1.5 Iab classification fully justified, but note the difficulty of distinguishing between M1.5 and M2.

We have one lucky-imaging epoch of $\alpha$ Sco. The WDS catalog contains data on the system that span a century and three quarters, but the dispersion is large, likely an effect of the large magnitude difference between $\mathrm{A}$ and $\mathrm{B}$. There is some evidence of a small inward and counterclockwise motion over that time span, but the period must be several thousand years at least.

\section{4. $\sigma$ Sco Aa,Ab, a spectral classification and extinction standard}

This is a well-known object that was used as one of the spectral classification standards for B1 III by Johnson \& Morgan (1953). More recently, it has also been used as an extinction standard because it is the prototype of $\sigma$-type sightlines (Krełowski et al. 1997; Cami et al. 1997; Maíz Apellániz 2015), in a similar way as $\zeta$ Oph is the prototype of $\zeta$-type sightlines. $\sigma$-type sightlines correspond to relatively lower density regions of the ISM that are exposed to UV radiation, while $\zeta$-type sightlines correspond to higher density regions of the ISM that are shielded from UV radiation. This division was initially applied to diffuse interstellar bands, but it has recently been discovered that a broad interstellar feature preferentially appears on $\sigma$-type sightlines (Maíz Apellániz et al. 2020a). There is no entry for this target in Gaia DR2, but from the HIPPARCos data and the Maíz Apellániz et al. (2008) prior, we derive a distance of $222_{-26}^{+34}$ pc. Alternatively, North et al. (2007) derived a distance of $174_{-18}^{+23} \mathrm{pc}$ from the Aa1,Aa2 orbit (see below).

$\sigma$ Sco has long been known to be a visual multiple. A faint companion (B) has recorded positions in the WDS catalog that date back to the eighteenth century. The Aa,Ab pair was discovered in the mid-twentieth century (Finsen 1956) with a $2.2 \mathrm{mag}$ difference and $d=00^{\prime \prime 3}-0^{\prime \prime} .5$. Finally, the Aa component is a close visual plus spectroscopic binary (Aa1,Aa2) with a $33 \mathrm{~d}$ eccentric orbit measured with interferometry and a 0.8 mag difference (North et al. 2007). North and collaborators estimated the spectral type of $\mathrm{Aa} 2$ to be $\mathrm{B} 1 \mathrm{~V}$ but noted that this is not a true spectral classification. Currently, Simbad gives as a spectral classification for $\sigma$ Sco $\mathrm{Aa}, \mathrm{Ab} \mathrm{O} 9.5+\mathrm{B} 7$, taken from Beavers \& Cook (1980), who attempted the spectral separation from an unresolved (either spatially or spectroscopically) spectrum of the system without acknowledging the existence of three components in the system. To our knowledge, no spatially resolved spectroscopy of $\mathrm{Aa}, \mathrm{Ab}$ exists in the literature.

We successfully spatially separated $\sigma$ Sco,Aa,Ab once with lucky spectroscopy (Fig. 4). The Aa component appears to be a single-lined B1 III object. We do not see double lines because the maximum velocity separation for the Aa1,Aa2 orbit is just $87 \mathrm{~km} \mathrm{~s}^{-1}$ (North et al. 2007), which is too low for a spectral resolution of 2500 . The $\mathrm{Ab}$ spectrum is relatively noisy, but we can constrain its spectral subtype by the absence of He II lines and the weakness of $\mathrm{Mg}$ II $\lambda 4481$ and its luminosity class by the width of its Balmer lines: it must be a B dwarf around B1. We clearly discard the B7 dwarf classification (or even any midB classification) by Beavers \& Cook (1980) because the Balmer lines are too narrow for this and $\mathrm{Mg}$ II $\lambda 4481$ is weak. The magnitude difference with Aa is compatible with the luminosity class difference at a similar spectral subtype especially when we consider that the difference between Aal and $\mathrm{Ab}$ is just 1.8 mag (accounting for the 0.8 mag between Aa1 and Aa2). Given the similar spectral types and relatively large magnitude difference between $\mathrm{Aa}$ and $\mathrm{Ab}$, no significant contribution from $\mathrm{Ab}$ is detected in the combined spectrum at the spectral resolution of 2500 .

We have one lucky-imaging epoch of $\sigma$ Sco, and the magnitude differences we measure are compatible with those in the WDS catalog. The WDS measurements of the Aa,Ab pair go back only to the 1970 s, but this is enough to reveal a significant clockwise motion with little variation in separation (possibly with a maximum around 2010). HIPPARCos measured $d=0$ ".428 and $\mathrm{PA}=58^{\circ}$ in 1991.25 , for instance. Assuming a circular faceon orbit, we obtain a period of 230-250 a.

There are 105 high-resolution epochs of $\sigma \mathrm{Sco}, \mathrm{Aa}, \mathrm{Ab}$ in LiLiMaRlin. Even though Aa1, Aa2, and Ab are inside the aperture, double lines are seen in many epochs but no triple lines, which is expected given the relatively small velocity amplitudes and how faint $\mathrm{Ab}$ is. In the future we plan to do spectral disentangling of the LiLiMaRlin data to confirm some of the results in this subsection.

\subsection{CS Cam $A, B$, a pair of luminous $B$ stars, one early and one late}

CS Cam A is a bright supergiant used by Morgan \& Roman (1950) as one of their B9 Ia standards. It has a B companion located 2 ".3 away and with a $3.6 \mathrm{mag}$ difference in the Tycho- 2 bands. The companion has mostly been ignored in the literature. The Gaia DR2 parallax for A combined with the Maíz Apellániz et al. (2008) prior and the previously used zeropoint leads to a distance of $813_{-138}^{+208} \mathrm{pc}$. The B component also has an entry in Gaia DR2, but its RUWE is larger than 1.4.

We successfully spatially separated CS Cam A,B twice with lucky spectroscopy. We show the results for one of the epochs in Fig. 5. The A component appears to be a B9 Ia, as expected from the literature. We classify the B component to be a B2 III, earlier and less luminous, which is its first-ever spectral classification. The combined spectrum shows no sign of the secondary, other than very slight changes in some He I lines that would easily remain unnoticed, at least at this spectral resolution.

There are 35 LiLiMaRlin epochs of CS Cam in which only the A component leaves a significant signal because B is not only considerably fainter, but also outside the aperture in most 


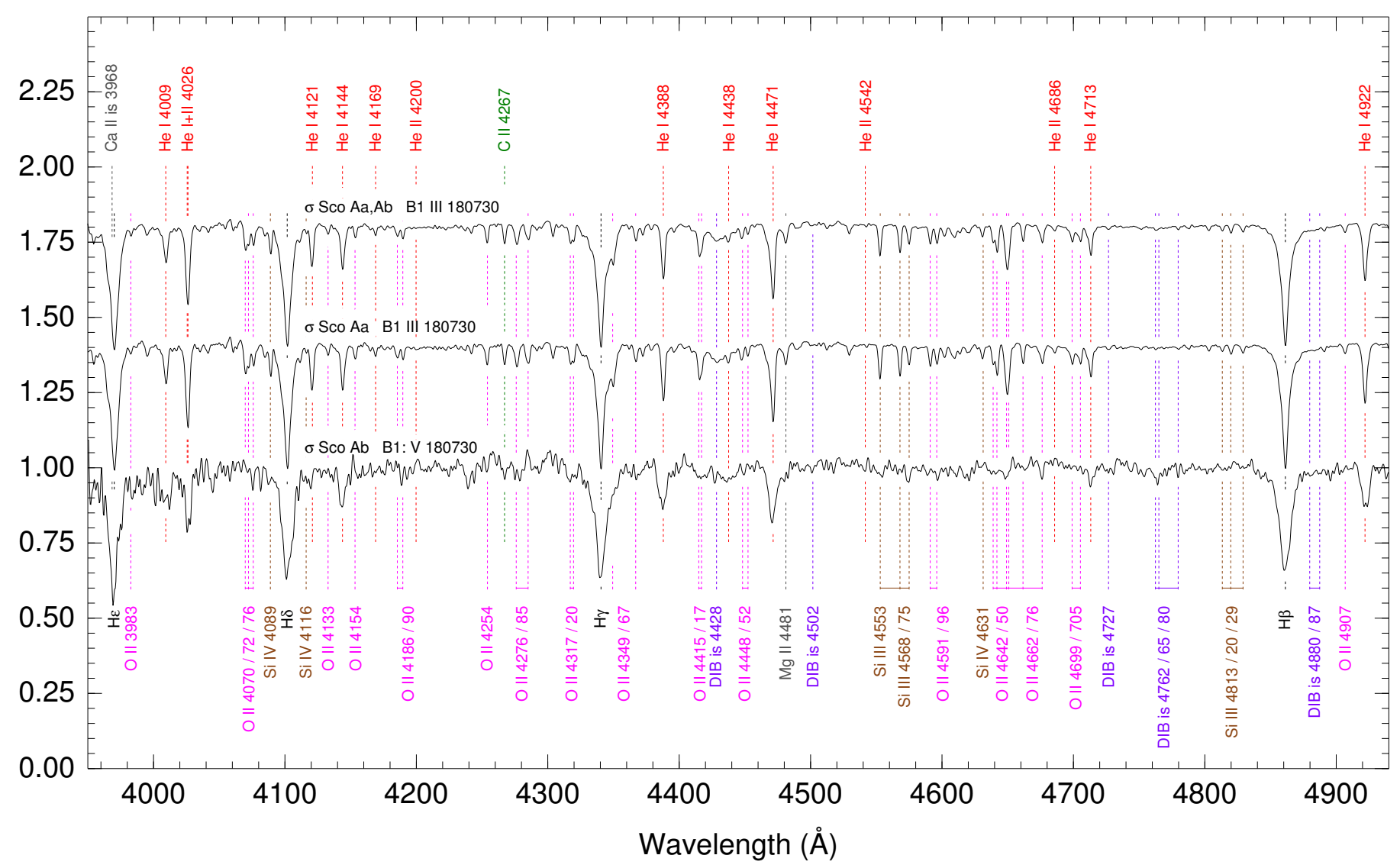

Fig. 4. Rectified spectrograms for $\sigma$ Sco at the GOSSS spectral resolution of $R \sim 2500$ and on the stellar reference frame. For each spectrogram, the name, spectral type, and evening date (YYMMDD) are shown. The top spectrogram is the weighted combination of the two components for the 180730 epoch. Main atomic and ISM lines are indicated.

cases. In the nine years covered by the observations no significant motion is recorded, but a velocity dispersion of $2-3 \mathrm{~km} \mathrm{~s}^{-1}$ is seen, which a common effect in B supergiants and is caused by pulsations (Simón-Díaz et al. 2020).

We have one lucky-imaging epoch of CS Cam. The magnitude differences in the $z$ and $Y$ bands are larger than in the HIPPARCOS $H$ band, which in turn are larger than in the Tycho-2 $B$ and $V$ bands, following the color differences between earlyand late-type B stars. The Gaia DR2 $G$ magnitude difference is larger but suspect, given the corrections associated with stars that are as bright as CS Cam A (future data releases may change this). The WDS catalog lists pair measurements that go back almost two centuries with no appreciable motion detected, but with considerable scatter in the oldest measurements. Both components appear in the Gaia DR2 catalog, and the astrometric data there are in excellent agreement with our measurements. On the other hand, Gaia DR2 detects a relative proper motion mostly in the inward direction that should have accumulated $\sim 0$ ". 4 over the two centuries of WDS observations. One way to reconcile the two results would be if the system had a near edge-on orbit that passed near quadrature about a century ago.

\section{6. $\sigma$ Ori $A a, A b, B$, an epoch with a better velocity separation}

This system has been previously extensively described in Simón-Díaz et al. (2015a), LS I, and MONOS I, to which we refer for information about it. We presented the first spatially resolved spectra of $\sigma$ Ori Aa,Ab and $\sigma$ Ori B in LS I. This observation took place on the night of 170907
$(\mathrm{HJD}=2458004.74)$, for which the spectroscopic orbit of Aa,Ab by Simón-Díaz et al. (2015a) predicts a velocity separation of just $15 \mathrm{~km} \mathrm{~s}^{-1}$, and we were understandably unable to kinematically separate the two components in the spectroscopic binary. In MONOS I we spatially separated the Aa, Ab and $\mathrm{B}$ components with a new lucky-spectroscopy observation obtained on the night of 181203 (HJD = 2458456.54$)$. The prediction for the velocity separation at that time was much more favourable, $234 \mathrm{~km} \mathrm{~s}^{-1}$, and indeed we were able to kinematically separate $\mathrm{Aa}$ and $\mathrm{Ab}$ and give spectral classifications for each. Here we present new spatially resolved spectra for the night of 190917 (HJD = 2458744.69$)$, where the predicted velocity separation was even larger, $288 \mathrm{~km} \mathrm{~s}^{-1}$, just $1 \mathrm{~km} \mathrm{~s}^{-1}$ shy of the maximum value (Fig. 6).

The new spectral classifications are the same as those in MONOS I, providing reassurance that when the velocity separation is large enough and lucky spectroscopy is performed under good seeing conditions, the result can be repeated. The spectral classification for the combined spectrum $A a, A b, B$ is the same as for $\mathrm{Aa}, \mathrm{Ab}$, as the large $v \sin i$ of $\mathrm{B}$ and the relative similarity between the three spectral types simply dilutes the lines. This point has been made before in Fig. 5 of Simón-Díaz et al. (2015a). The good velocity separation between Aa and Ab allows us to make a precise measurement of the flux ratio between the two of $0.70 \pm 0.05$ or about $0.4 \mathrm{mag}$, which is higher than the value in Simón-Díaz et al. (2015a), but not by much. We also note that the latest spectrogram of $\sigma$ Ori B might alternatively be interpreted as a partially resolved SB2 of two similar stars, given the asymmetry of some lines such as He I $\lambda 4471$, 


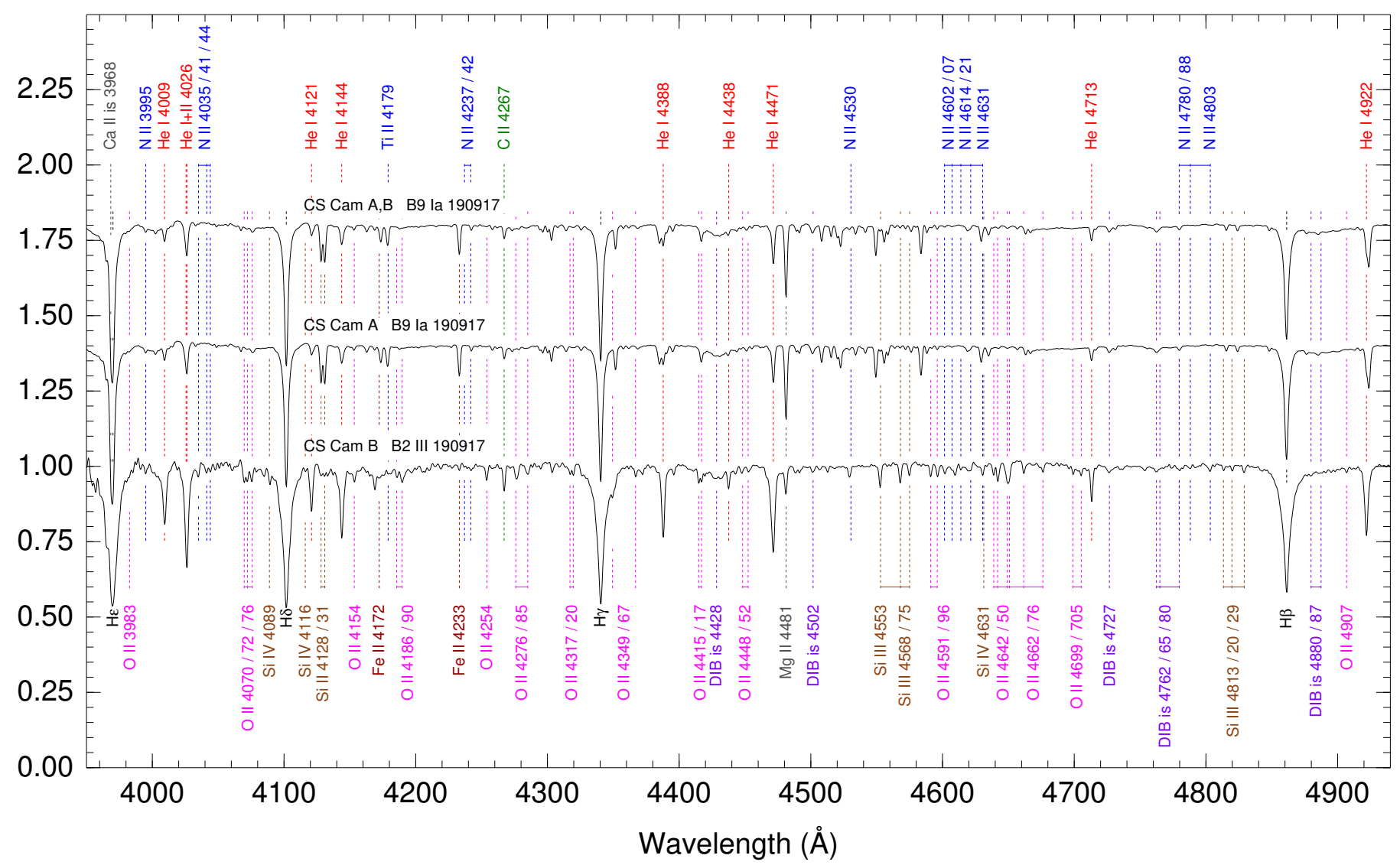

Fig. 5. Rectified spectrograms for CS Cam at the GOSSS spectral resolution of $R \sim 2500$ and on the stellar reference frame. For each spectrogram, the name, spectral type, and evening date (YYMMDD) are shown. The top spectrogram is the weighted combination of the two components for the 190917 epoch. Main atomic and ISM lines are indicated.

which would make the $\sigma$ Ori system an SB2+SB2. However, the effect may not be real but be the effect of pulsations or of noise in the spatial disentangling. Further observations may solve the issue, possibly lucky spectroscopy at other phases to detect a larger velocity amplitude in the alleged SB2 or interferometry to spatially resolve it.

\subsection{HD 5005 A,B,C,D, a trapezium system with an early-type $O$ star}

HD 5005 is a Trapezium system at the core of the IC 1590 cluster that ionizes the NGC $281 \mathrm{H}$ II region, also known as the Pacman nebula. In GOSSS I we classified its four main components (A, B, C, and D) as O stars. In the case of A and B, we based the spectral classifications on a spatial disentangling of the two components, which have $d=1 " \prime 6$ and $\Delta m=1.6 \mathrm{mag}$. All four main components have Gaia DR2 entries with good values of RUWE, so that we can combine them in the same way as we did for $6 \mathrm{Cas}$ to obtain a distance to HD 5005 of $2.53_{-0.27}^{+0.33} \mathrm{kpc}$.

We used lucky spectroscopy to spatially separate HD 5005 A,B in two epochs (Fig. 7). The two epochs yield the same spectral classification for the A component, O4.5 V $((\mathrm{fc})) \mathrm{z}$, similar to that of GOSSS I, but later by half a spectral subtype and with the $\mathrm{z}$ suffix that indicates that He II $\lambda 4686$ is deeper than in normal stars with V luminosity class. For the B component we derive B0 V for both epochs, but with an uncertain spectral subtype for the 190906 epoch because of the lower $\mathrm{S} / \mathrm{N}$ that is generated by the spatial disentangling. This spectral classification is later than the O9.5 $\mathrm{V}$ from GOSSS $\mathrm{I}$ and moves HD $5005 \mathrm{~B}$ from the
O-star to the B-star category. This is a good example of how lucky spectroscopy allows a cleaner separation of the spectra of close binaries than the traditional long-exposure methods.

The first lucky-spectroscopy epoch (181219) was obtained with the QUCAM3 setup and the second epoch (190906) with the EEV12 setup. The HD 5005 B QUCAM3 setup has a better $\mathrm{S} / \mathrm{N}$, as we suspected from the comparison between the two setups, confirming that it is preferred to the EEV12 epoch for faint stars. Nevertheless, both setups produce valid and consistent results.

The A,B spectrum in Fig. 7 is of pedagogical value. When the two components (an $\mathrm{O} 4.5$ and a B0) are combined, we obtain an $\mathrm{O} 6 \mathrm{~V}((\mathrm{f}))$ star with no major abnormalities. For example, the C III $\lambda \lambda \lambda 4647,4650,4651$ triplet is in weak emission in A (hence, the c suffix) and in strong absorption in B. When these two are combined (weighted by the $\Delta m$ ), the feature disappears, as expected for a normal O6 dwarf. On the other hand, the N III $\lambda \lambda \lambda 4634,4641,4642$ triplet, also in emission in $\mathrm{A}$ and in absorption in $\mathrm{B}$, is left in emission in the combined spectrum (as expected for an object with an ((f)) suffix) because they have similar strengths in each component and the magnitude difference allows the emission to dominate. Therefore it is possible for such an unresolved binary system to remain undetected in single-epoch spectroscopy with $R \sim 2500$ and to make us believe that the object is a single star of intermediate spectral type.

There are nine LiLiMaRlin epochs of HD 5005 A,B, but the data are hard to interpret because the separation between the two components combined with the different apertures for each spectrograph and seeing variations make the contribution 


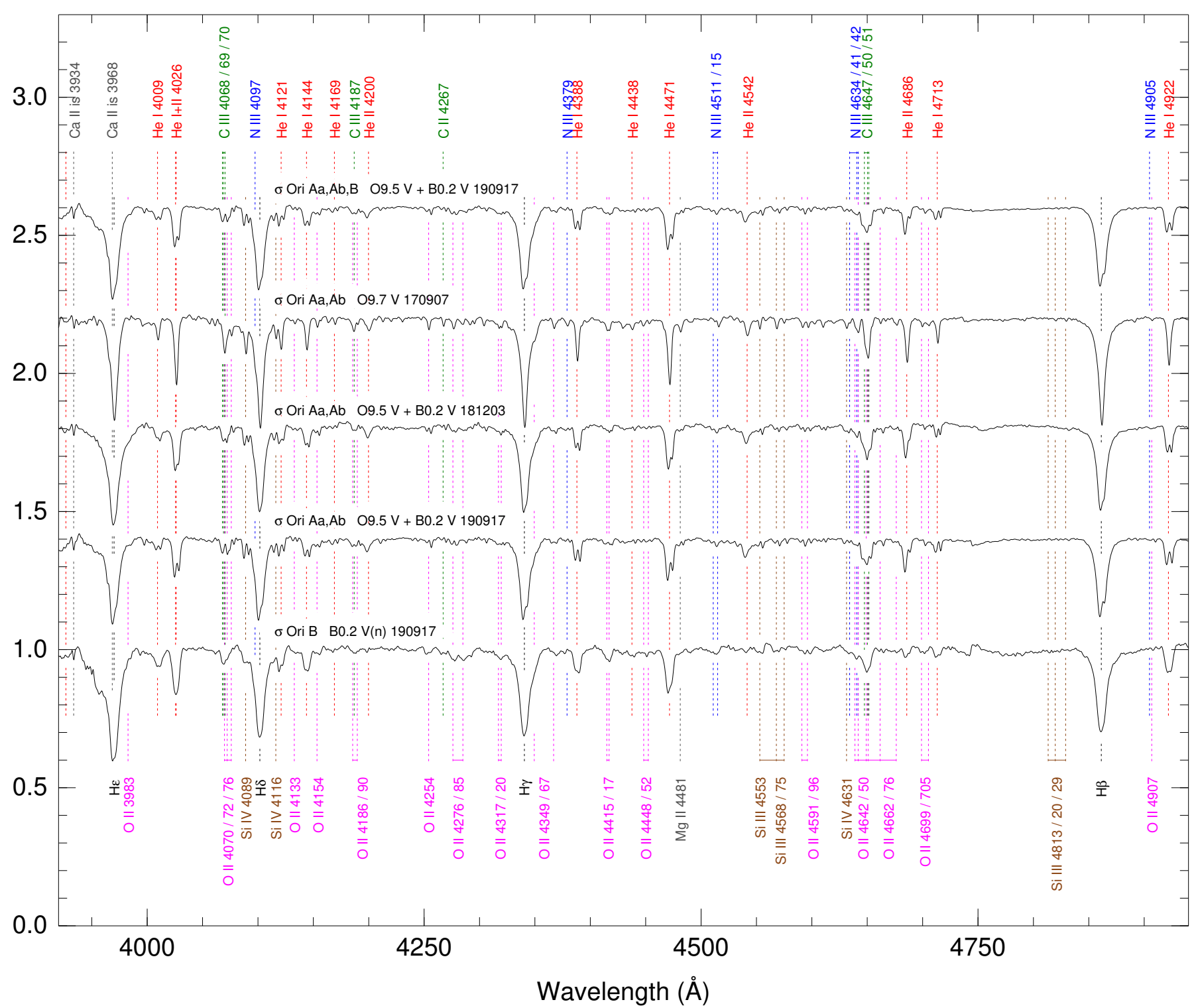

Fig. 6. Rectified spectrograms for $\sigma$ Ori at the GOSSS spectral resolution of $R \sim 2500$ and on the heliocentric reference frame. For each spectrogram, the name, spectral type, and evening date (YYMMDD) are shown. The top spectrogram is the weighted combination of the two components for the 190917 epoch. Main atomic and ISM lines are indicated.

of each of the two components different in each epoch. The He II lines, dominated by A, are relatively stable in intensity (and also in velocity, indicating the absence of a spectroscopic companion). On the other hand, He I $\lambda 4922$, dominated by the B component (Fig. 7), has large artificial variations in intensity caused by this effect. This is a good example for the necessity of spatially resolved spectroscopy in studying spectroscopic binarity. This system is also of particular interest because it is one of the cases in which quantitative spectroscopy causes the early-O star to lie closer to the ZAMS than the bulk of Galactic O-type stars analyzed by Holgado et al. (2020). It is of prime importance to determine to which extent the $\mathrm{B}$ companion affects the derived parameters to ascertain the reliability of the position of this star in the spectroscopic HR diagram.

For HD 5005 we have three good-quality AstraLux epochs, one of which has been reported in Maíz Apellániz (2010). They span a period of 11 years. There is no appreciable motion between the A and B components in this time span. This is consistent with the WDS data, which go back a century and a half with nearly constant position angles and separations and with the relative proper motion in Gaia DR2, which is essentially zero in right ascension and within two sigmas of this value in declination.

\subsection{HD $51756 A, B$, a system of two similar components with a new $O$ star}

HD 51756 is a bright but relatively poorly studied early-type system with spectral classifications in Simbad that range in spectral subtype from B0 to B3 and in luminosity class from IV to Ib. It is composed of a pair A,B separated by 0, . 7 and with a small magnitude difference of just 0.3 mag. A third $\mathrm{C}$ component is dimmer and located $13^{\prime \prime}$ away. The A,B pair has no entries in Gaia DR2, but the C component has a parallax of $0.5398 \pm 0.0575$ mas and a good RUWE there. Using the Gaia DR2 for the C component as we did previously for FN CMa, we obtain a distance of $1.79_{-0.17}^{+0.21} \mathrm{kpc}$. 


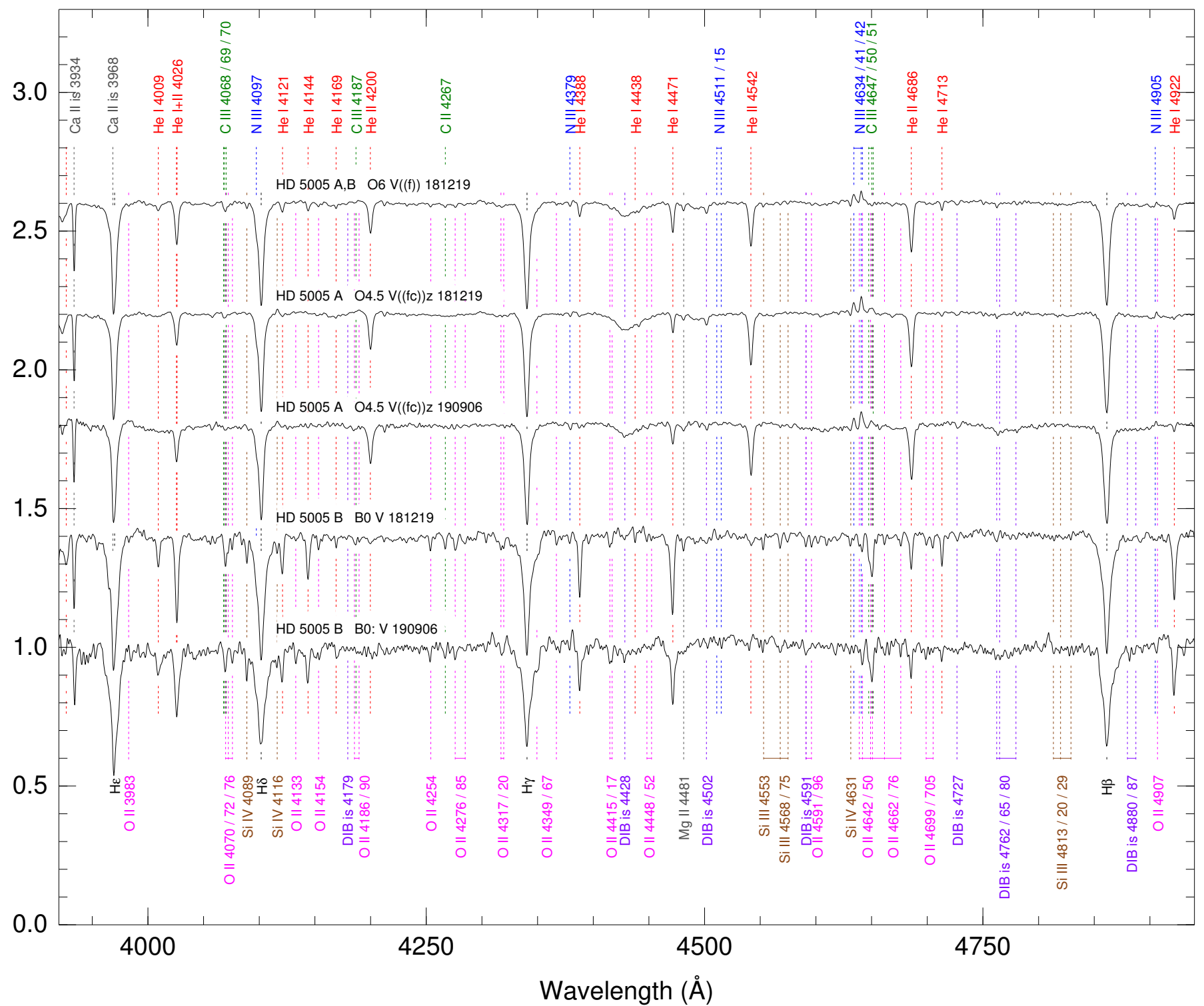

Fig. 7. Rectified spectrograms for HD 5005 at the GOSSS spectral resolution of $R \sim 2500$ and on the stellar reference frame. For each spectrogram, the name, spectral type, and evening date (YYMMDD) are shown. The top spectrogram is the weighted combination of the two components for the 181219 epoch. Main atomic and ISM lines are indicated.

We spatially resolved the A and B components with lucky spectroscopy (Fig. 8), and we derived classifications of B0 IV for the first (brighter) component and O9.7 IV(n) for the second (dimmer) component. The two spectral classifications are similar, but B is a faster rotator and of an earlier subtype. This is the first time that HD $51756 \mathrm{~B}$ has been identified as an $\mathrm{O}$ star, and given its magnitude $(V \sim 8.1 \mathrm{mag})$, it is one of the brightest objects of that type identified recently. Roman-Lopes et al. (2018) have indicated that the composite A,B spectrum is O9.7 III. For the composite, we obtain B0 IV instead (Fig. 8); the small difference being likely caused by not matching spectral resolutions between standards and observations, which is a large effect around O9.7/B0 spectral subtypes caused by the differences in intrinsic line width between He II $\lambda 4542$ and Si III $\lambda 4552$. This system is similar to FN CMa in that the $\mathrm{B}$ star in an $\mathrm{O}+\mathrm{B}$ pair is capable of hiding the nature of the hotter companion in the unresolved spectrum.

There are 18 LiLiMaRlin epochs for HD 51756 A,B, where both visual components are included. The line profiles at high resolution clearly show a composite profile, with a narrow and a broad component, to which we assign A and B, respectively, based on our lucky spectroscopy. The narrow component shows velocity variations with a peak-to-peak amplitude of $\sim 20 \mathrm{~km} \mathrm{~s}^{-1}$, suggesting that $A$ is an SB1. If that were the case, it would explain why A is slightly brighter than B even though it is onequarter of a spectral subtype later and of the same luminosity class because the second star (which would be Ab if it were resolved) would contribute to the light without leaving a significant signature in the spectrum (possibly because it is a fast rotator, as B is; this would make this system quite similar to $\sigma$ Ori).

We have two lucky-imaging epochs for this system, and our results agree with those of HIPPARCOS from 1991.25 in terms of separation, position angle, and magnitude difference (there are no entries for this system in Gaia DR2). The only slight difference is in position angle, for which there is a small counterclockwise movement that is also consistent with the historical WDS data that stretch back a century and a half. For a circular face-on orbit the corresponding period is about $7 \mathrm{ka}$. 


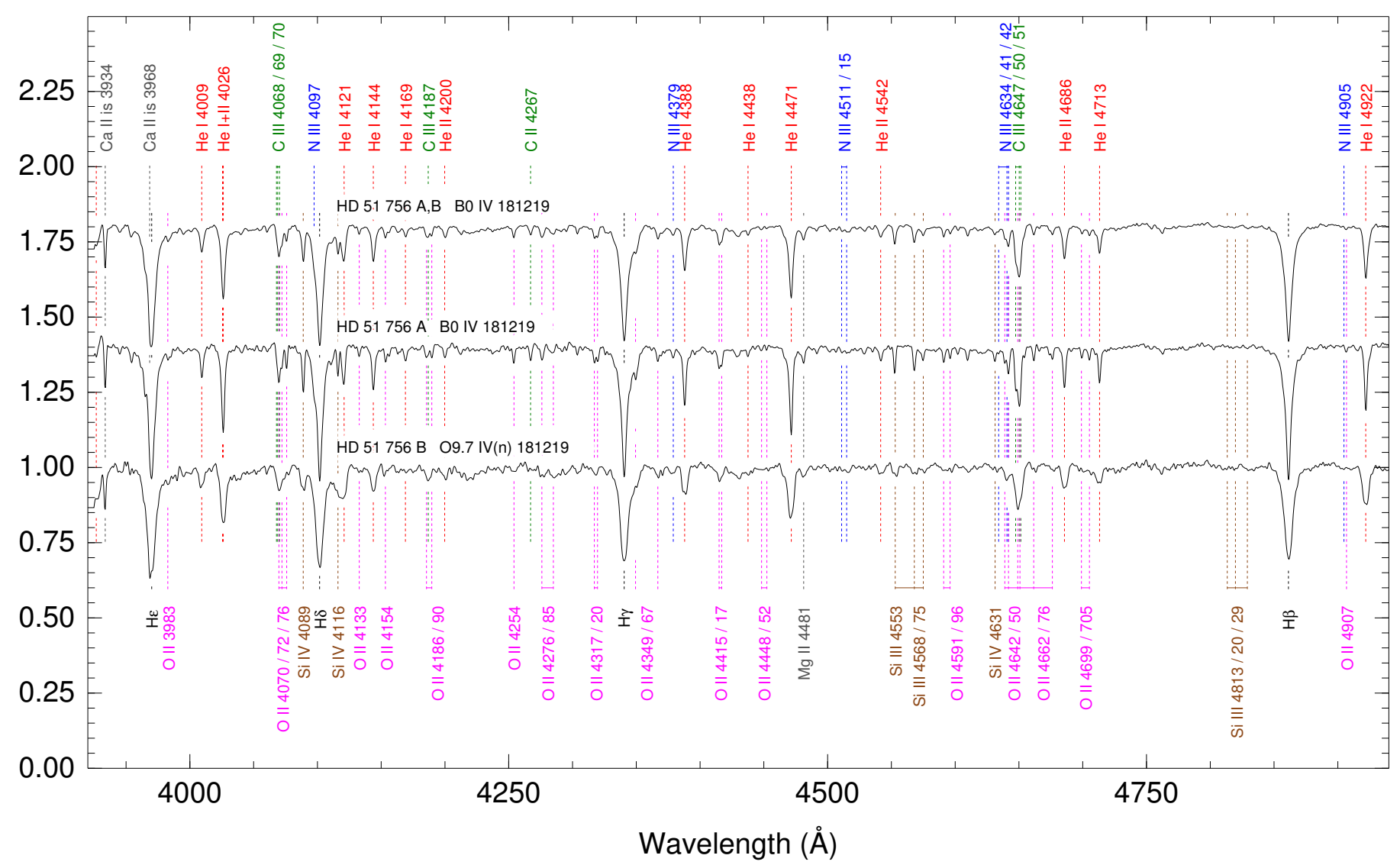

Fig. 8. Rectified spectrograms for HD 51756 at the GOSSS spectral resolution of $R \sim 2500$ and on the stellar reference frame. For each spectrogram, the name, spectral type, and evening date (YYMMDD) are shown. The top spectrogram is the weighted combination of the two components for the 181219 epoch. Main atomic and ISM lines are indicated.

\section{9. $H D 218195 A, B$, a system with an N-rich primary}

HD $218195 \mathrm{~A}$ is a nitrogen-enhanced O star (GOSSS II) with a B component located at a separation of 0 '.94 that is 2.7 mag dimmer. The WDS catalog lists two weaker components, C and D, farther away. The A component has an entry in Gaia DR2, but with a RUWE of 1.67, so that we cannot use its parallax to derive the distance. There is no clearly defined cluster around the object, and the C component (Gaia DR2 2010239999586616448) also has a high RUWE. Our last resort to obtain a Gaia DR2 distance to the system is to assume that the D component (Gaia DR2 2010239999586619392, with a RUWE of 0.94 ), located $11^{\prime \prime} 4$ away, is physically bound to A,B. If this is the case, the distance to the system is $2.29_{-0.17}^{+0.20} \mathrm{kpc}$.

Despite the large magnitude difference, we were able to spatially separate A and B with lucky spectroscopy and obtain a clean spectrum of the weak component. HD 218195 A maintains the O8.5 IIINstr classification of GOSSS II (Fig. 9) because the B component exerts little effect on the combined spectrum at $R \sim 2500$ (but see below for the high-resolution case). The B component, which has been identified as an early-B star in GOSSS I, has a spectral type of B1.5 V. As an indication of the clean separation of the two components, the B spectrum has no anomalies around the wavelengths where He II lines are seen in the A spectrum.

The eight LiLiMaRlin epochs for HD 218195 A,B span 14 years. Both components are included in the aperture. All the strong lines appear to be single, and there are no appreciable velocity variations, indicating that the primary has no spectroscopic companions. However, the situation changes for the weak lines, in which the contribution of the $\mathrm{B}$ component dominates despite the large magnitude difference, such as C II $\lambda 4267$ and some O II lines. These lines show significant time-dependent changes, and at least one epoch shows double profiles with a velocity separation of $\sim 70 \mathrm{~km} \mathrm{~s}^{-1}$. We also note that some lines in the B spectrum in Fig. 9 are slightly asymmetric. Therefore we tentatively identify the B component as an SB2.

We have two lucky-imaging epochs for this system; one has been reported in Maíz Apellániz (2010). Our results are in reasonable agreement with those of HIPPARCos from 1991.25 in terms of separation, position angle, and magnitude difference. There are a few historical observations of this pair in the WDS catalog, but the scarce data from the early twentieth century indicate a small secular increase in separation.

\subsection{HD 219460 A,WR 157, disentangling two very different spectra}

HD 219460 is a visual binary whose A component is of B spectral type. Its B component is a Wolf-Rayet (WR) star (WR 157). The system was classified as B1 II + WN4.5 by Turner et al. (1983) based on an spatially unresolved spectrum, which is feasible because of the very different ionic species and absorption or emission nature of the lines present in an early-B supergiant and a WR star (Fig. 10). The separation between the two components is 1 ".37 and the magnitude difference is small, with A being 0.4 mag brighter than B in Tycho-2 B. Both HD 219460 A 


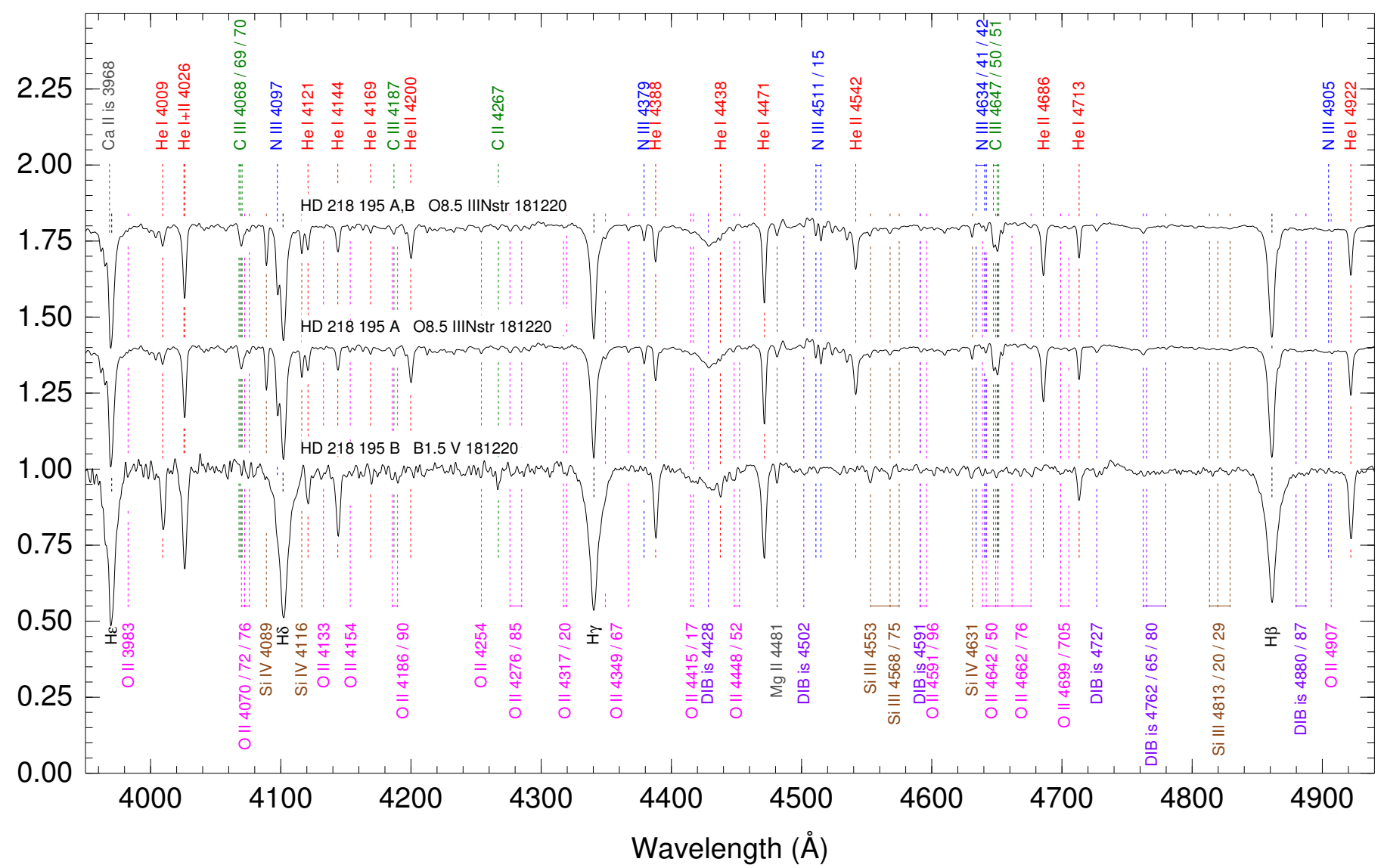

Fig. 9. Rectified spectrograms for HD 218195 at the GOSSS spectral resolution of $R \sim 2500$ and on the stellar reference frame. For each spectrogram, the name, spectral type, and evening date (YYMMDD) are shown. The top spectrogram is the weighted combination of the two components for the 181220 epoch. Main atomic and ISM lines are indicated.

and WR 157 have Gaia DR2 entries, but the RUWE for the latter is 1.9 , prompting us to use the former to calculate the distance. Using the same parallax zeropoint and prior as before, we obtain $2.58_{-0.21}^{+0.25} \mathrm{kpc}$.

Given the small magnitude difference and the relatively large separation, this system is relatively easy to spatially separate with lucky spectroscopy. We point out, however, how clean the disentangling is in Fig. 10: there are no signs of emission lines in the B star, and the only absorption lines in the WR star are all caused by the ISM. The only exception are the absorption components in the P-Cygni profiles for N V $\lambda \lambda 4604,4620$. The absorption lines for the A component are asymmetric. Based on the narrowest lines (e.g., He I 25016 ), we identify the system as an SB2 caught with a velocity of $\sim 175 \mathrm{~km} \mathrm{~s}^{-1}$. The primary spectroscopic component can be accurately classified as B0.7 II (quite similar to the Turner et al. 1983 result), but the spectral type of the secondary spectroscopic component is more uncertain. Further epochs are necessary to determine whether the system can be caught with a larger velocity difference. The B component (WR 157) is classified as WN5 according to the criteria for nitrogen lines in Smith et al. (1996) because our spectrum does not reach to He II $\lambda 5412$ or He I $\lambda 5876$. The composite spectrum in Fig. 10 shows the (diluted) combination of the two visual components.

For this system we have two lucky-imaging epochs. Our position angles and separations are very similar and are in excellent agreement with those of Gaia DR2 because both components are also detected there. Our magnitude differences are negative, meaning that WR 157 is brighter than HD $219460 \mathrm{~A}$ in our bands. The magnitude difference decreases from Tycho-2 $B$ to Tycho-2 $V$ to Gaia DR2 (all positive) to $i$ to $z$ (both negative), indicating that the B component is redder than the A component. The Gaia DR2 relative proper motion should lead to a counterclockwise motion of WR 157 with respect to HD 219460 A of $\sim 6^{\circ}$ between the early twentieth century and today, but no such effect is seen in the average of the two epochs around that time in the WDS catalog.

\subsection{1. $H D 8768 A, B$, an $O C+B$ visual binary}

HD 8768 was classified as O9.5 IV by Morgan et al. (1955), who were unaware of the presence of a B component with $d=0$ ".66 and $\Delta m=2.6$ mag (Maíz Apellániz 2010). A third C component is located 5'.9 away but is too faint to make a significant contribution to the spectrum. This object has received little attention in the literature compared to other $\mathrm{O}$ stars of similar magnitude. The pair is unresolved in Gaia DR2 and has a negative parallax and a RUWE value of 3.2, likely as a result of the different contributions from each component to each observation. The $\mathrm{C}$ component, on the other hand, has a valid RUWE, and we can use it to estimate the distance (assuming it is physically associated with the A,B pair) with the same parallax zeropoint and prior as before to obtain $3.11_{-0.52}^{+0.77} \mathrm{kpc}$.

We spatially separated the A and B components with lucky spectroscopy (Fig. 11). For A we obtain a spectral classification of OC9.2 II, as established by its weak nitrogen lines (e.g., N III $\lambda \lambda 4511,4515)$ and strong carbon lines (e.g., C III $\lambda \lambda \lambda 4068,4069,4070)$, adding in this way a new member to 


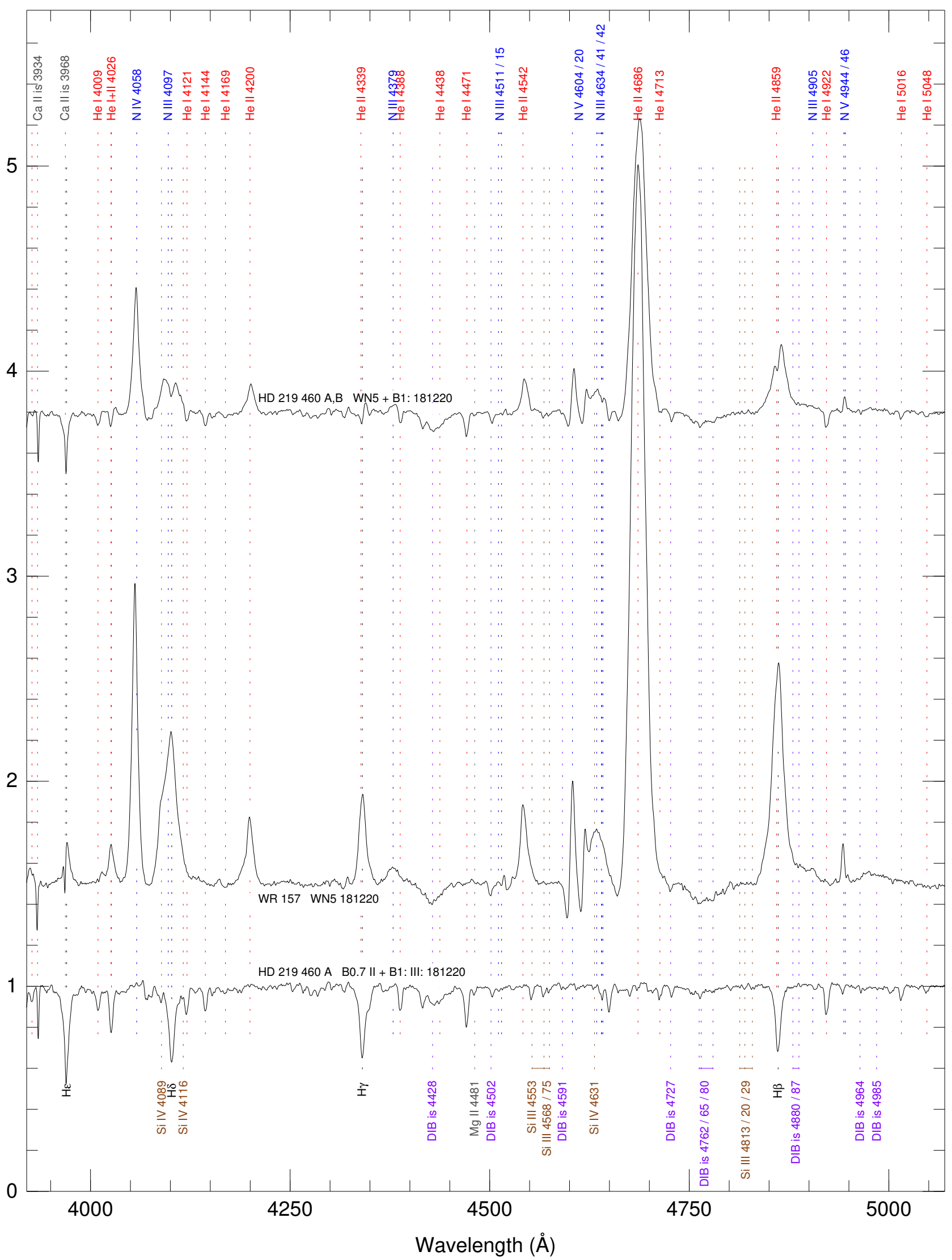

Fig. 10. Rectified spectrograms for HD 219460 at the GOSSS spectral resolution of $R \sim 2500$ and on the stellar reference frame. For each spectrogram, the name, spectral type, and evening date (YYMMDD) are shown. The top spectrogram is the weighted combination of the two components for the 181220 epoch. Main atomic and ISM lines are indicated. 


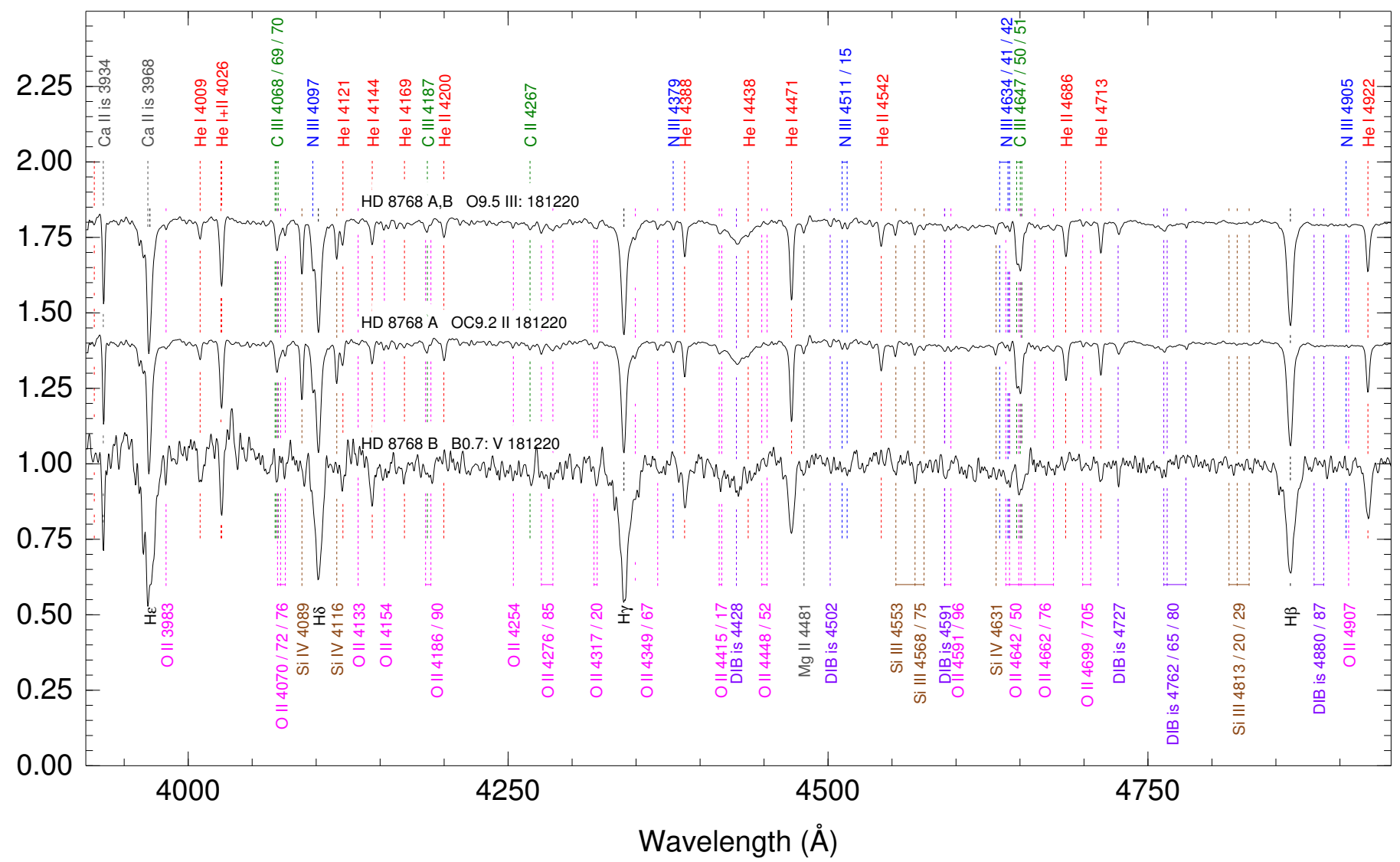

Fig. 11. Rectified spectrograms for HD 8768 at the GOSSS spectral resolution of $R \sim 2500$ and on the stellar reference frame. For each spectrogram, the name, spectral type, and evening date (YYMMDD) are shown. The top spectrogram is the weighted combination of the two components for the 181220 epoch. Main atomic and ISM lines are indicated.

the rare class of carbon-enriched $\mathrm{O}$ stars. The spectrogram for $\mathrm{B}$ is noisy as a consequence of the small separation and large magnitude difference, but we are able to establish that it is an early-B dwarf with a most likely subtype of B0.7. The combined spectrum shows a small effect of the B component in moving the spectral subtype to O9.5 and leaving an uncertain luminosity classification due to the inconsistency between different classification criteria (a problem common to such combinations of lateO + early-B binaries, see Simón-Díaz et al. 2015a). This effect explains the original classification by Morgan et al. (1955).

We have three lucky-imaging epochs of this system, one of which has been reported in Maíz Apellániz (2010). This and the HIPPARCos data point are the only data in the WDS catalog, so there is little historical information. Our data show an indication of a counterclockwise motion of B around A, but the uncertainties are large, therefore new epochs are needed to confirm it. The motion corresponds to a period of at least several thousand years.

\subsection{2. $B D+60544 A, B$, a new O-type system}

This system has no previous accurate spectral classifications listed in Simbad, just two references that indicate that it is of OB type (Hardorp et al. 1959; Rydstrom 1978). The WDS catalog indicates that it is a pair separated by 1.9 with a $\Delta m$ of 0.9 mag and a first measurement from a century ago. Both components have entries with good RUWE in Gaia DR2 and with parallaxes within one sigma of each other. We combined the two with the same zeropoint and prior as before (and including the covariance term) to obtain a distance of $2.24_{-0.25}^{+0.33} \mathrm{kpc}$.
This system is relatively easy to spatially separate with lucky spectroscopy given its relatively small magnitude difference and large separation (Fig. 12). The A component is classified as O9.5 IV, making this the first time that it is identified as an $\mathrm{O}$ star. The B component, on the other hand, is classified as $\mathrm{B} 1 \mathrm{~V}$. The merged spectrum receives the intermediate classification of O9.7 IV:, with the uncertainty in the luminosity class being another instance of contradicting luminosity criteria when a late-O star and an early-B star are combined.

We have one lucky-imaging epoch of BD +60 544. Both components are also detected in Gaia DR2, and our values for the separation, position angle, and magnitude difference agree well.

\subsection{3. $H D 17520 A, B$, a system with an Oe star}

This system was analyzed in previous GOSSS papers, and in GOSSS III, the A component was assigned a spectral type of $\mathrm{O} 8 \mathrm{~V}$ and the B component a spectral type of O9: Ve. The system has one of the smallest separations in this paper $\left(00^{\prime \prime} 32\right)$; its magnitude difference is $0.7 \mathrm{mag}$. These classifications were made with regular long-slit spectroscopy. A reanalysis of the previous data has revealed that we erroneously identified the two components by reversing the position of the slit: the component with the Oe spectrum is actually A, not B. HD 17520 A,B has a single entry in Gaia DR2, but with a poor RUWE and a negative parallax. The $\mathrm{C}$ component, on the other hand, has a valid RUWE, and we can use it to estimate the distance (assuming it is physically associated with A,B) with the same parallax zeropoint and 


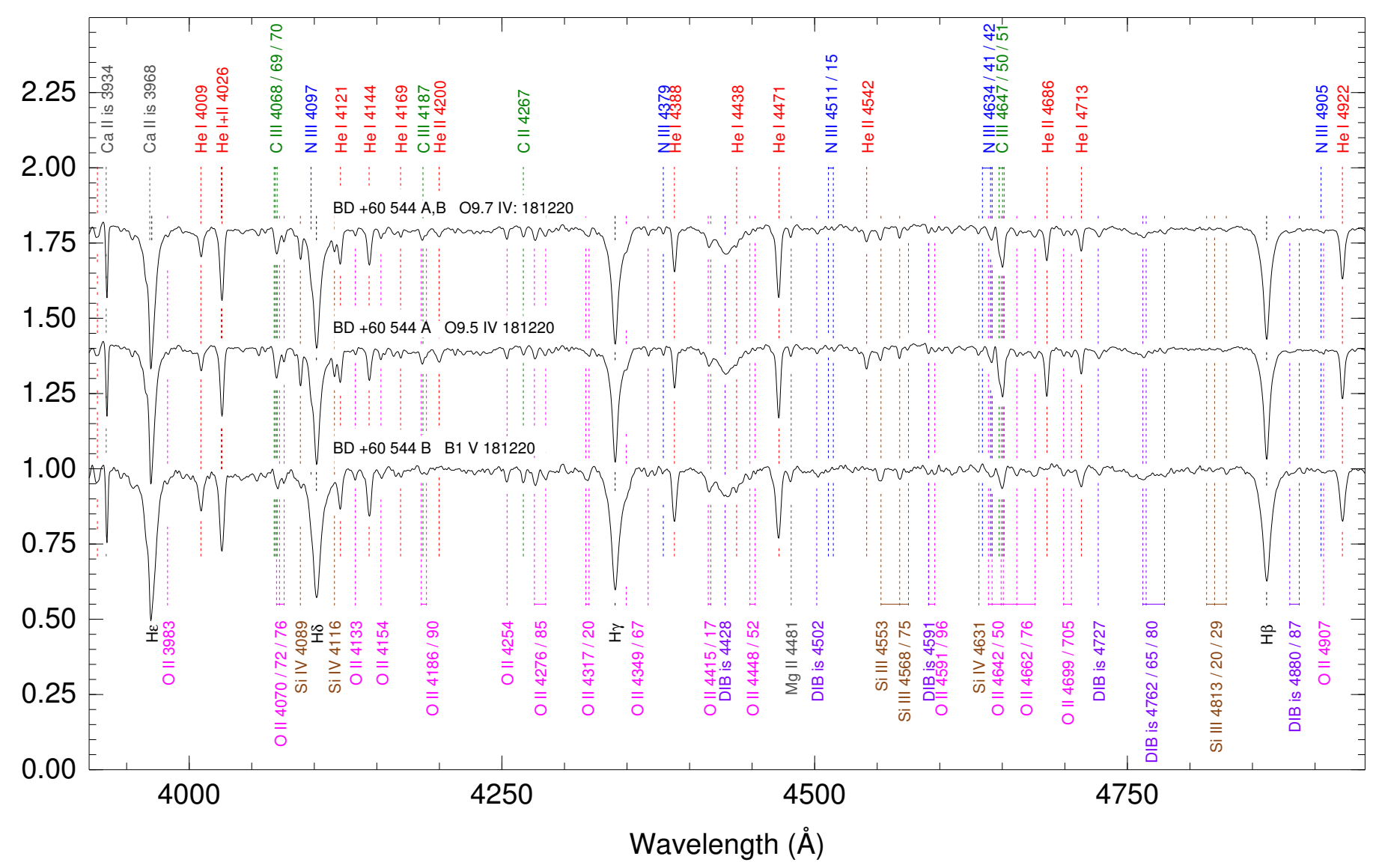

Fig. 12. Rectified spectrograms for BD +60544 at the GOSSS spectral resolution of $R \sim 2500$ and on the stellar reference frame. For each spectrogram, the name, spectral type, and evening date (YYMMDD) are shown. The top spectrogram is the weighted combination of the two components for the 181220 epoch. Main atomic and ISM lines are indicated.

prior as before to obtain $2.69_{-0.26}^{+0.32} \mathrm{kpc}$. HD 17520 is in IC 1848 , whose characteristics derived from Gaia data will be analyzed in more detail in a subsequent paper of our Villafranca series of Galactic groups with OB stars (Maíz Apellániz et al. 2020b).

Despite the small magnitude difference, we are able to clearly separate the two components with lucky spectroscopy (Fig. 13), as shown by the opposite behavior of $\mathrm{H} \beta$ in $\mathrm{A}$ and $\mathrm{B}$. The A component has a spectral classification of $09.2 \mathrm{Ve}$ and the $\mathrm{B}$ component one of $\mathrm{O} 8 \mathrm{Vz}$. The new classifications are similar to those previously obtained in GOSSS, but the A and B components are exchanged and significantly cleaner at the same time. The merged spectrum is classified as $08.5 \mathrm{~V}$ with a $\mathrm{p}$ suffix to indicate discrepancies between weak lines caused by the composite nature. The double-peaked emission profile in $\mathrm{H} \beta$ characteristic of high-inclination $\mathrm{Oe} / \mathrm{Be}$ stars is strong in $\mathrm{A}$, absent in $\mathrm{B}$, and weak in the merged spectrum.

There are 16 LiLiMaRlin epochs of HD 17520 A,B, and the most notorious feature is that the double-peaked emission in $\mathrm{H} \beta$ and the even stronger one in $\mathrm{H} \alpha$ experience not only the flux changes typical in Oe/Be stars, but also large velocity variations with a peak-to-peak amplitude of $\sim 200 \mathrm{~km} \mathrm{~s}^{-1}$. This indicates that the A component is an SB1 and explains why it is brighter than B although it is of a later subtype. We plan to obtain additional lucky-spectroscopy epochs to attempt catching $\mathrm{A}$ at a more favourable velocity separation and possibly identify the missing spectroscopic component.

We have two lucky-imaging epochs for this system, one of which has been reported in Maíz Apellániz (2010). The combination of our results and the historical WDS data indicates a small counterclockwise and outward motion of B with respect to A. The corresponding period would be of several thousand years.

\subsection{HD $24431 A, B$, a nearby isolated system}

HD 24431 was classified as O9 III in Walborn (1973) and received the same classification in GOSSS I. The WDS cata$\log$ lists a B component with $d=0, .7$ and $\Delta m=2.9$ mag. There is a single Gaia DR2 entry for this system, but it has a RUWE of 1.69 , likely caused by the B component. The target is isolated, and we have been unable to find a stellar group associated with it in the Gaia DR2 data, leaving us no alternative but to use the Gaia DR2 parallax to estimate a distance of $812_{-53}^{+61} \mathrm{pc}$, where the uncertainty is likely an underestimation because of the high RUWE. Nevertheless, we point out that HIPPARCos also obtained a high value of the parallax for HD 24431 (with a much larger uncertainty) and that the spectroscopic parallax of Maíz Apellániz \& Barbá (2018) is $\sim 1$ kpc, so that this system appears to be relatively nearby compared to most $\mathrm{O}$ stars in the sample of this paper.

We were able to separate A and B with lucky spectroscopy (Fig. 14). The spectral classification of $A$ (and of the merged spectrum, where B contributes little) is the same as the previous classification from GOSSS I. The spectrum of B is somewhat noisy, but not as much as that of HD $8768 \mathrm{~B}$, for example, which has a similar separation and magnitude difference with respect to its primary (but which is fainter in absolute terms). Its spectral classification is B1.5 V. 


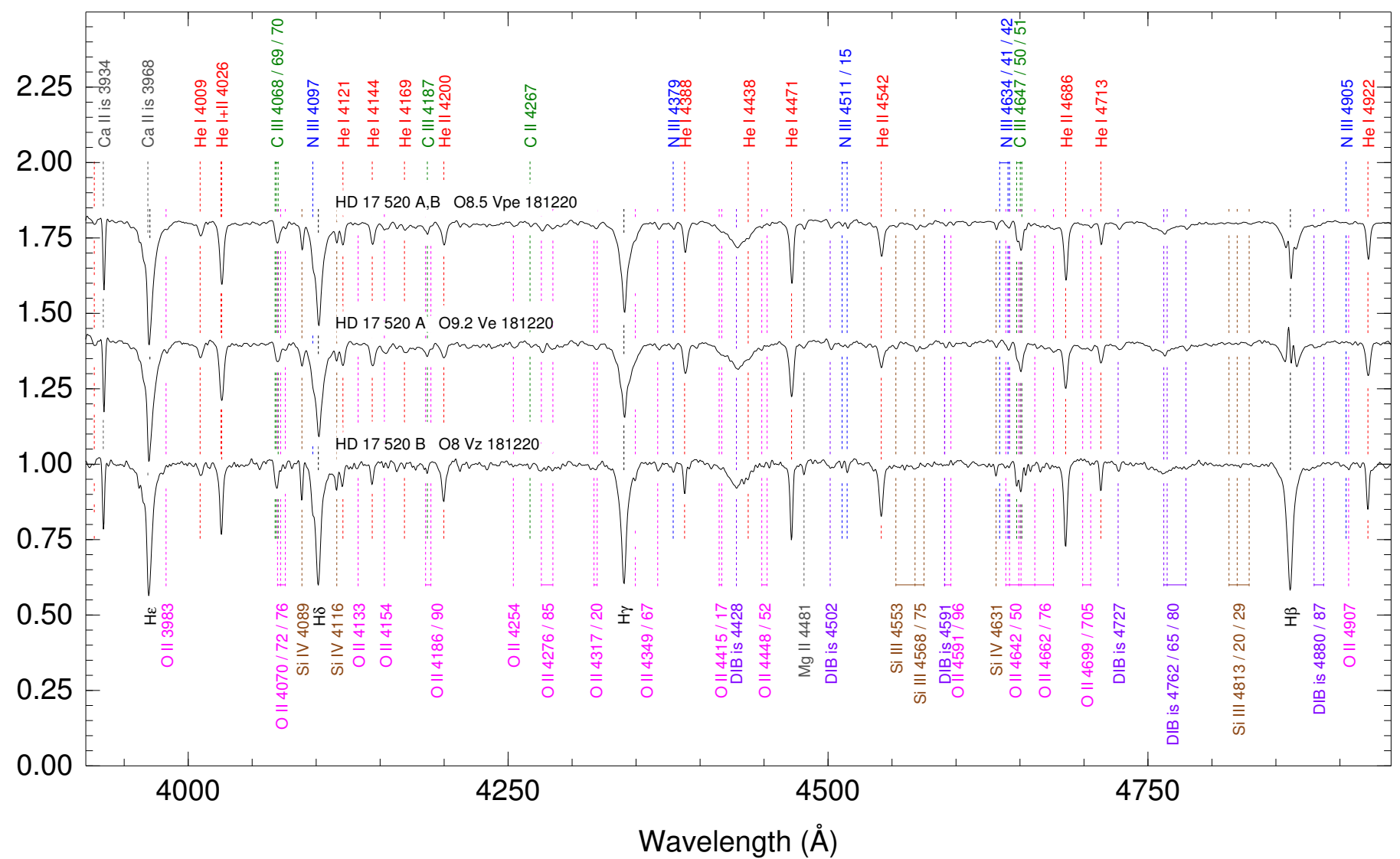

Fig. 13. Rectified spectrograms for HD 17520 at the GOSSS spectral resolution of $R \sim 2500$ and on the stellar reference frame. For each spectrogram, the name, spectral type, and evening date (YYMMDD) are shown. The top spectrogram is the weighted combination of the two components for the 181220 epoch. Main atomic and ISM lines are indicated.

We have 22 LiLiMaRlin epochs, where the light from both components is included, but the contribution from A is clearly dominant. HD 24431 A appears as an SB1 in the high-resolution spectra with a peak-to-peak amplitude of $\sim 50 \mathrm{~km} \mathrm{~s}^{-1}$. To our knowledge, this is the first identification of the target as an SB1.

For this system we have two lucky-imaging epochs, one of which has been reported in Maíz Apellániz (2010). The combination of our results and the WDS data that starts with the HIPPARCos measurements shows a clockwise orbital motion of $\mathrm{B}$ with respect to A. For a face-on circular motion, the estimated period is $4-5 \mathrm{ka}$.

\subsection{HD $164492 A, B$, an O star with a misclassified companion}

HD 164492 is the high-order multiple system at the center of M20, also known as NGC 6514 or as the Trifid nebula. The A component is a well-known 07.5 Vz star (GOSSS II), and the WDS catalog lists another ten components, making this a borderline case between a high-order multiple system and a small cluster (considering the likelihood of additional low-mass stars hiding in the glare of the high- and intermediate-mass stars and the existence of other components that are unaccounted for in the catalog, see below). A itself is listed as an Aa,Ab pair, but $\mathrm{Ab}$ is just 25 mas away from $\mathrm{Aa}$ and is a red object with a $\Delta H$ of 3 magnitudes (Sana et al. 2014), so its contribution in the optical is negligible for the purposes of this paper. The second brightest component is $\mathrm{C}$, which itself is an interesting triple system composed of a static, fast-rotating, magnetic early-B star (C1) and a spectroscopic pair $(\mathrm{C} 2+\mathrm{C} 3)$ of two narrow-lined early- and lateB stars (Wade et al. 2017; González et al. 2017).

Simbad currently gives a spectral classification of A2 Ia for the B component that can be traced back to Gahm et al. (1983) and that is problematic for several reasons. The first reason is that it implies the coexistence in an $\mathrm{H}$ II region of a very young O7.5 Vz star and an evolved A supergiant, which under normal circumstances would require a significantly older age. The second reason is that an A supergiant should be significantly brighter in the optical and in the near-IR than an O7.5 Vz star and the WDS catalog lists the A component being almost 3 magnitudes brighter than B (see also below). This would require the A supergiant either being significantly obscured by differential extinction and/or being much farther away than the remaining stars. The first option is excluded by the 2MASS colors and the second by the Gaia DR2 parallaxes (see below).

To estimate the distance to HD 164492 we followed a strategy similar to the one we used for 6 Cas, combining Gaia DR2 entries with good RUWE and similar parallaxes and proper motions, but selecting among the bright named WDS components. We excluded $C$ due to its large RUWE (see above for its multiplicity, the likely reason) and we kept the other four bright components (Table 5). Applying the same parallax zeropoint and prior as before, we obtain a distance of $1.29_{-0.10}^{+0.12} \mathrm{kpc}$. We note that the proper motions indicate significant internal motions, as expected for a high-order multiple system such as this.

We placed the slit along the A-B direction and easily separated the two components with lucky spectroscopy (Fig. 15), as expected given the large separation $\left(6^{\prime \prime} \cdot 26\right)$. The A and the 


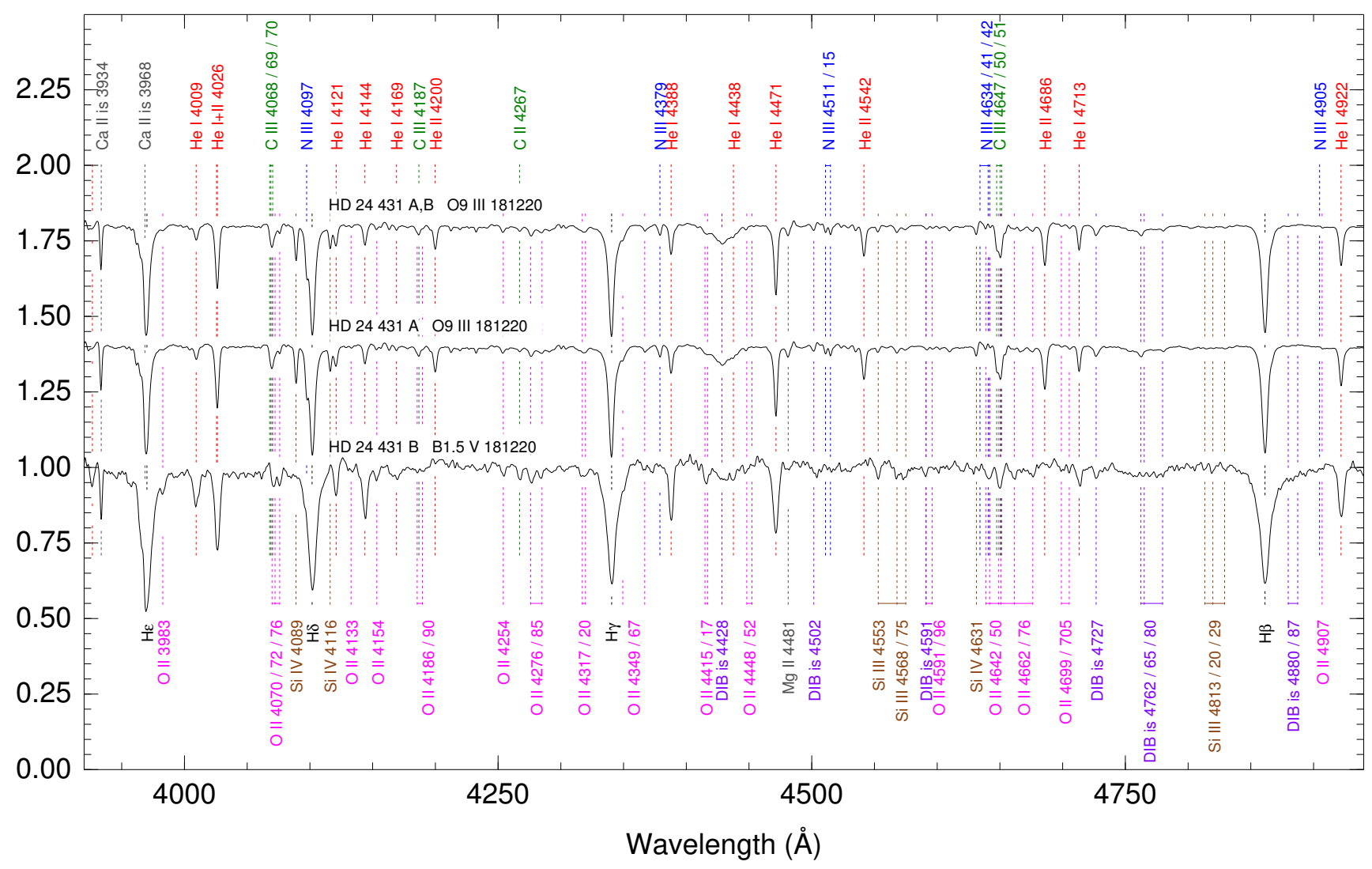

Fig. 14. Rectified spectrograms for HD 24431 at the GOSSS spectral resolution of $R \sim 2500$ and on the stellar reference frame. For each spectrogram, the name, spectral type, and evening date (YYMMDD) are shown. The top spectrogram is the weighted combination of the two components for the 181220 epoch. Main atomic and ISM lines are indicated.

Table 5. Gaia DR2 astrometric data for four components of the HD 164492 system with compatible parallaxes and proper motions and their aggregate results.

\begin{tabular}{lccc}
\hline \hline Comp. & $\begin{array}{c}\varpi \\
\text { (mas) }\end{array}$ & $\begin{array}{c}\mu_{\alpha *} \\
(\mathrm{mas} / \mathrm{a})\end{array}$ & $\begin{array}{c}\mu_{\delta} \\
(\mathrm{mas} / \mathrm{a})\end{array}$ \\
\hline $\mathrm{A}$ & $0.6376 \pm 0.0873$ & $0.426 \pm 0.122$ & $-1.765 \pm 0.097$ \\
$\mathrm{~B}$ & $0.7780 \pm 0.0757$ & $0.308 \pm 0.106$ & $-1.196 \pm 0.083$ \\
$\mathrm{D}$ & $0.8049 \pm 0.1071$ & $1.055 \pm 0.140$ & $-2.858 \pm 0.112$ \\
$\mathrm{E}$ & $0.8793 \pm 0.0685$ & $0.842 \pm 0.093$ & $-1.548 \pm 0.073$ \\
\hline & $0.7841 \pm 0.0613$ & $0.630 \pm 0.084$ & $-1.706 \pm 0.082$ \\
\hline
\end{tabular}

Notes. The aggregate results use external uncertainties and include the spatial covariance terms of Lindegren et al. (2018).

merged A,B spectra receive the same spectral classification as in GOSSS II (the B component is too weak to make a significant dent in the merged spectrum). The B component is a B2 Vnn star, that is, not an A supergiant, but instead a mid-B star with a very large $v \sin i$ that makes it a peculiar object, but not for the reason given by Gahm et al. (1983). Our spectral classification for HD 164492 B suggests possible explanations for the previous A supergiant classification: the object could be a pre-mainsequence star (PMS) that was observed by Gahm et al. (1983) while it was undergoing a shell phase, or it could be a Be star currently in a phase with a weak disk. We also observed the $\mathrm{C}$ component with standard long-slit spectroscopy and assign it a spectral classification of B1 V, which must be dominated by the C2 component of Wade et al. (2017) because the C1 component is easily hidden by its large $v \sin i$ and the C 3 component is significantly fainter.

There are 12 epochs of HD 164492 A in LiLiMaRlin spanning 13 years. No changes in the spectral appearance or in velocity can be appreciated in the data. There is also one FEROS epoch of HD 164492 B (possible given the large separation) that is consistent with our lucky-spectroscopy observation. We have used this epoch to measure the $v \sin i$ of this fast rotator as we did for $\alpha$ Sco B (which has a quite similar spectrum), in this case using five He I lines: He I $\lambda 4387$, He I $\lambda 4471, \mathrm{He}$ I $\lambda 4713$, He I $\lambda 5876$, and He I $\lambda 6678$. We obtain a value of $355 \pm 55 \mathrm{~km} \mathrm{~s}^{-1}$. Finally, there are also 20 epochs of HD $164492 \mathrm{C}$ in LiLiMaRlin spanning 6 years that show the complex line profiles produced by the SB3 system.

We have a single lucky-imaging epoch for HD 164492 . In this case, we list in Table 3 the measurements for three of the pairs. We did not attempt to resolve $\mathrm{A}$ into $\mathrm{Aa}+\mathrm{Ab}$ becuse the predicted separation is too small for AstraLux. Our data for the A,B and A,C pairs agree reasonably well with the Gaia DR2 results. For the A,H pair our magnitude difference is compatible with that of Turner et al. (2008), obtained at similar wavelengths, but is significantly larger than the near-IR values of Sana et al. (2014), indicating that $\mathrm{H}$ is redder than $\mathrm{A}$.

\subsection{6. $H D 168021 A, B, C$, a triple system of early- $B$ supergiants}

This system is a hierarchical triple composed of a close pair $(\mathrm{A}, \mathrm{B})$ separated by 0.48 and with a $\Delta m$ of $1.0 \mathrm{mag}$ and a more distant companion (C) $17^{\prime \prime} \cdot 2$ away and with a magnitude 


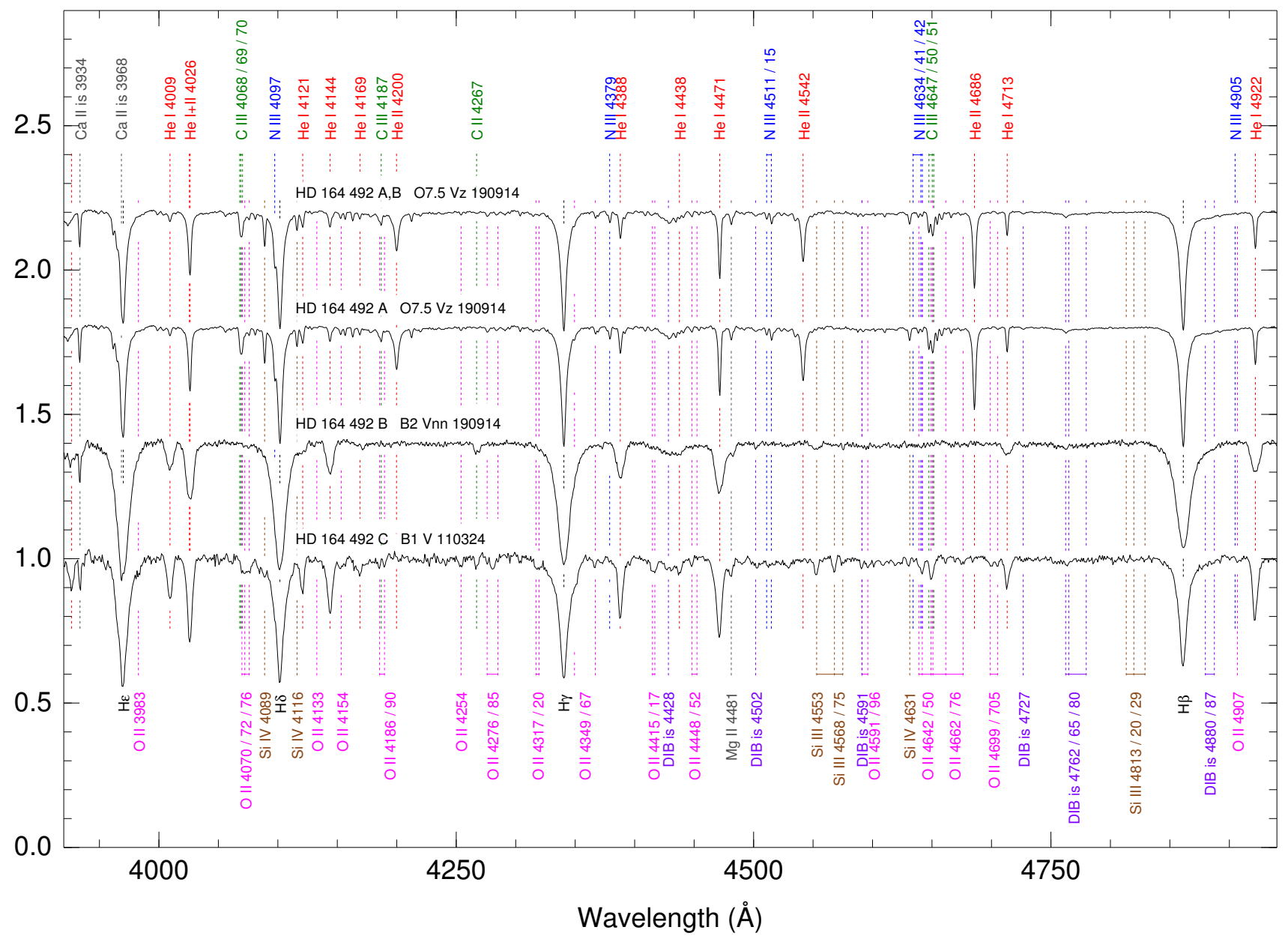

Fig. 15. Rectified spectrograms for HD 164492 at the GOSSS spectral resolution of $R \sim 2500$ and on the stellar reference frame. For each spectrogram, the name, spectral type, and evening date (YYMMDD) are shown. The 110324 data were obtained with the 2.5 duPont telescope at LCO i.e. not with lucky spectroscopy. The top spectrogram is the weighted combination of the two components for the 190914 epoch. Main atomic and ISM lines are indicated.

intermediate between those of $\mathrm{A}$ and $\mathrm{B}$, according to the WDS catalog. Morgan et al. (1953) classified the unresolved A,B pair as $\mathrm{B} 0 \mathrm{Ib}$. The $\mathrm{C}$ component has no precise spectral classification in the literature. There is a single Gaia DR2 entry for the A,B pair, but it has a poor RUWE, so that we cannot use it to estimate the distance to the system. HD $168021 \mathrm{C}$, on the other hand, has an entry with a good RUWE that allows us to obtain a value of $1.62_{-0.14}^{+0.18} \mathrm{kpc}$.

We spatially resolve A and B with lucky spectroscopy (Fig. 16), and we find that both are relatively similar earlyB supergiants. The A component is earlier and more luminous at B0 Ia, while the B component is later and less luminous at B0.2 II. The combined spectrum is classified as B0 Iab, that is, the same spectral subtype as in the Morgan et al. (1953) classification, but changing the luminosity class from Ib to Iab. We also observed the $\mathrm{C}$ component with the WHT using regular long-slit spectroscopy and determined a spectral classification of B0.5 II. According to this, HD 168021 includes three similar early-type B supergiants.

\subsection{HD $193443 A, B$, comparing STIS and lucky spectroscopy}

HD 193443 is a visual double separated by 0 "'.138 and with a small magnitude difference ( $B$ is actually slightly brighter than A) that was studied in STIS I. In that paper, spectral classifications of O8.5 III((f)) and O9.2 IV were given for A and B, respectively. One of the components is a spectroscopic binary (Mahy et al. 2013), and in STIS I we favoured that it is B but stated that more observations are needed to confirm this hypothesis. There is a single entry in Gaia DR2 for HD 193443 A,B, but we cannot use it for a distance determination because its RUWE is poor. The WDS catalog lists a C component 9'.1 away, but its RUWE is also poor. The only way to give a Gaia DR2 distance to the system therefore is by including it in the Cyg OB1 association, for which Ward et al. (2020) give a value of $1.64 \pm 0.24 \mathrm{kpc}$. We note that Simbad currently assigns a spectral classification of $09.5 \mathrm{~V}$ to HD $193443 \mathrm{C}$ and cites Mahy et al. (2015). This is a case of mistaken identity in Simbad because that paper refers to the spectroscopic binary, not to a visual companion. The $\mathrm{C}$ component is too faint to be an $\mathrm{O}$ star at the same distance as the A,B pair.

In the sample in STIS I, HD 193443 A,B is one of the two easiest systems to separate with the HST (see Fig. 4 in that paper). For this reason, we attempted to separate the pair with lucky spectroscopy, and we were able to do so on an occasion with exceptional seeing (we also attempted to separate the other easy STIS system, HD 16429 Aa,Ab, but we were unsuccessful). We compare in Fig. 17 the spectra from both setups. The spectra for each component are compatible, but the lucky-spectroscopy results are much noisier, illustrating the 


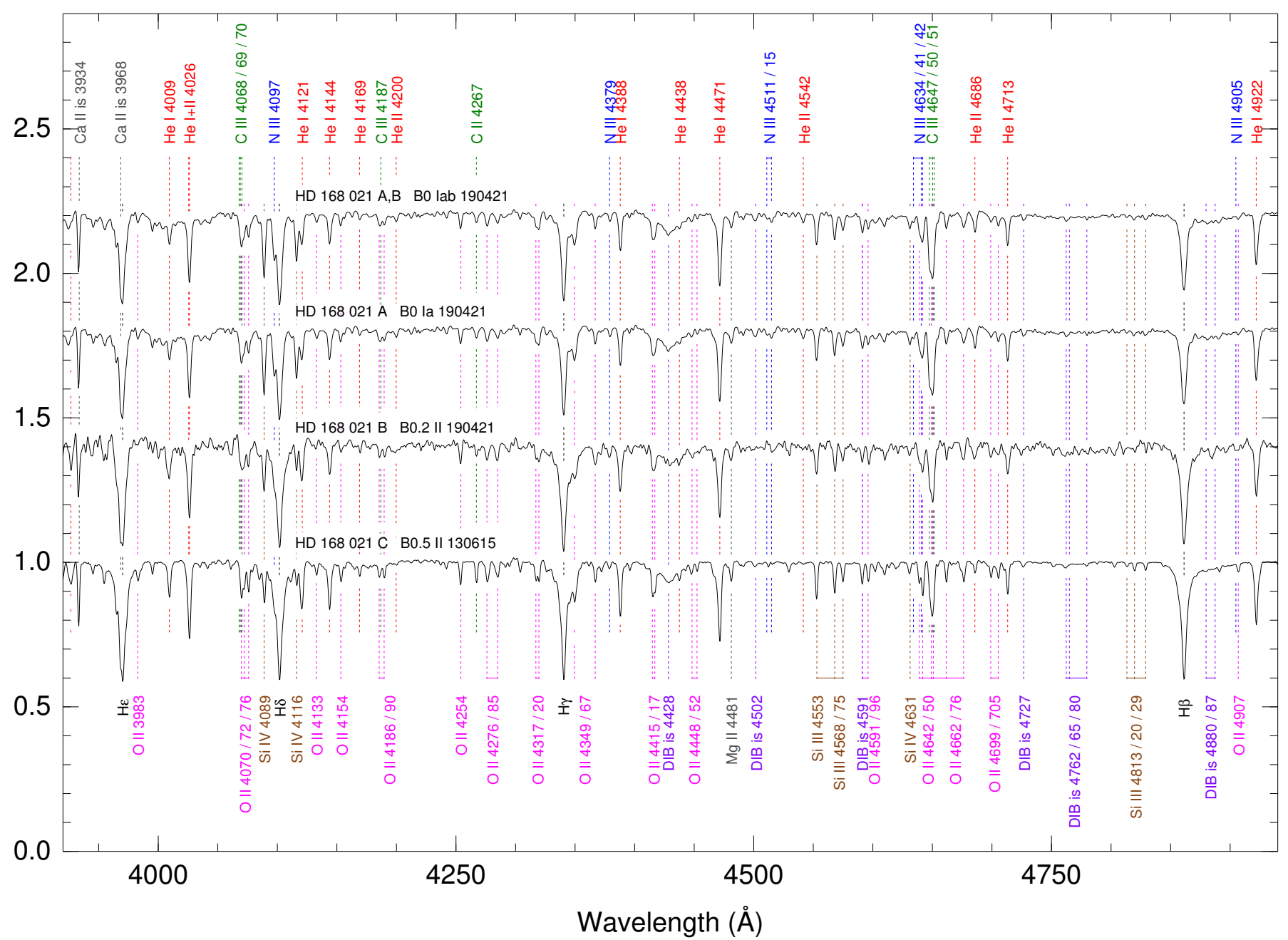

Fig. 16. Rectified spectrograms for HD 168021 at the GOSSS spectral resolution of $R \sim 2500$ and on the stellar reference frame. For each spectrogram, the name, spectral type, and evening date (YYMMDD) are shown. The 130615 data were obtained with WHT but not with lucky spectroscopy. The top spectrogram is the weighted combination of the two components for the 190421 epoch. Main atomic and ISM lines are indicated.

different spatial resolving power of HST and lucky spectroscopy. This translates into uncertain spectral types derived from the ground-based data.

We have 29 LiLiMaRlin epochs of HD 193443 A,B that show a clear signal of the spectroscopic motion of different components. However, as already mentioned in STIS I, this is an SB3 with a static component (likely A) and two objects (likely two stars in B) that move in a spectroscopic orbit with a period of $7.467 \mathrm{~d}$ and a relatively low velocity amplitude $\left(K_{1}+K_{2}\right.$ $\sim 130 \mathrm{~km} \mathrm{~s}^{-1}$, Mahy et al. 2013), which is not large enough to make the three components appear separated at any point of the orbit. In future papers we will analyse this system in more detail with the help of new data.

\subsection{HD $194649 A, B$, identifying the SB2 in a visual binary}

This system was included in MONOS I, where we presented spatially unresolved spectroscopy and lucky-imaging results. It is a pair with $\Delta m=0.95 \mathrm{mag}$ (with a possible color term) and $d=0$ ".40. Similarly to HD 193443 , it includes a spectroscopic binary with a 3.39294 d period (Mahy et al. 2013), but previous results were not able to spatially separate the A and
B visual components. In MONOS I we derived spectral classifications of O6 $\mathrm{V}((\mathrm{f}))+\mathrm{O} 9.7$ : $\mathrm{V}$ with GOSSS data and of O6 IV((f)) + O9.5 V((f)) with LiLiMaRlin data, but we acknowledged that three stars contribute to the spectra and that further studies of the system are therefore required. HD 194649 has a single entry in Gaia DR2, but without parallax or proper motions due to the extremely high RUWE of the solution, 108.1, caused by the lack of resolution of the system into its two visual components. The system is the brightest object of a little-studied stellar cluster listed in Simbad as [KPS2012] MWSC 3335 (Kharchenko et al. 2013). We have searched Gaia DR2 to select the brightest members with good RUWE according to their proper motions and calculate the distance to the cluster (Table 6), obtaining a result of $1.62_{-0.10}^{+0.12} \mathrm{kpc}$.

The two visual components in HD 194649 are clearly resolved with lucky spectroscopy (Fig. 18). The SB2 in the system is the A component, with a spectral classification of O5.5 V $((\mathrm{f}))+09.5 \mathrm{~V}$. The B component, on the other hand, is classified as $\mathrm{O} 7 \mathrm{Vz}$. The merged spectrum appears as O6 V $((f))+$ O9.7: V, the same classification we obtained with GOSSS data in MONOS I. These results paint a coherent picture in which the late-O star (the one with the largest velocity amplitude in the SB2) is separated in velocity in the merged spectrum 


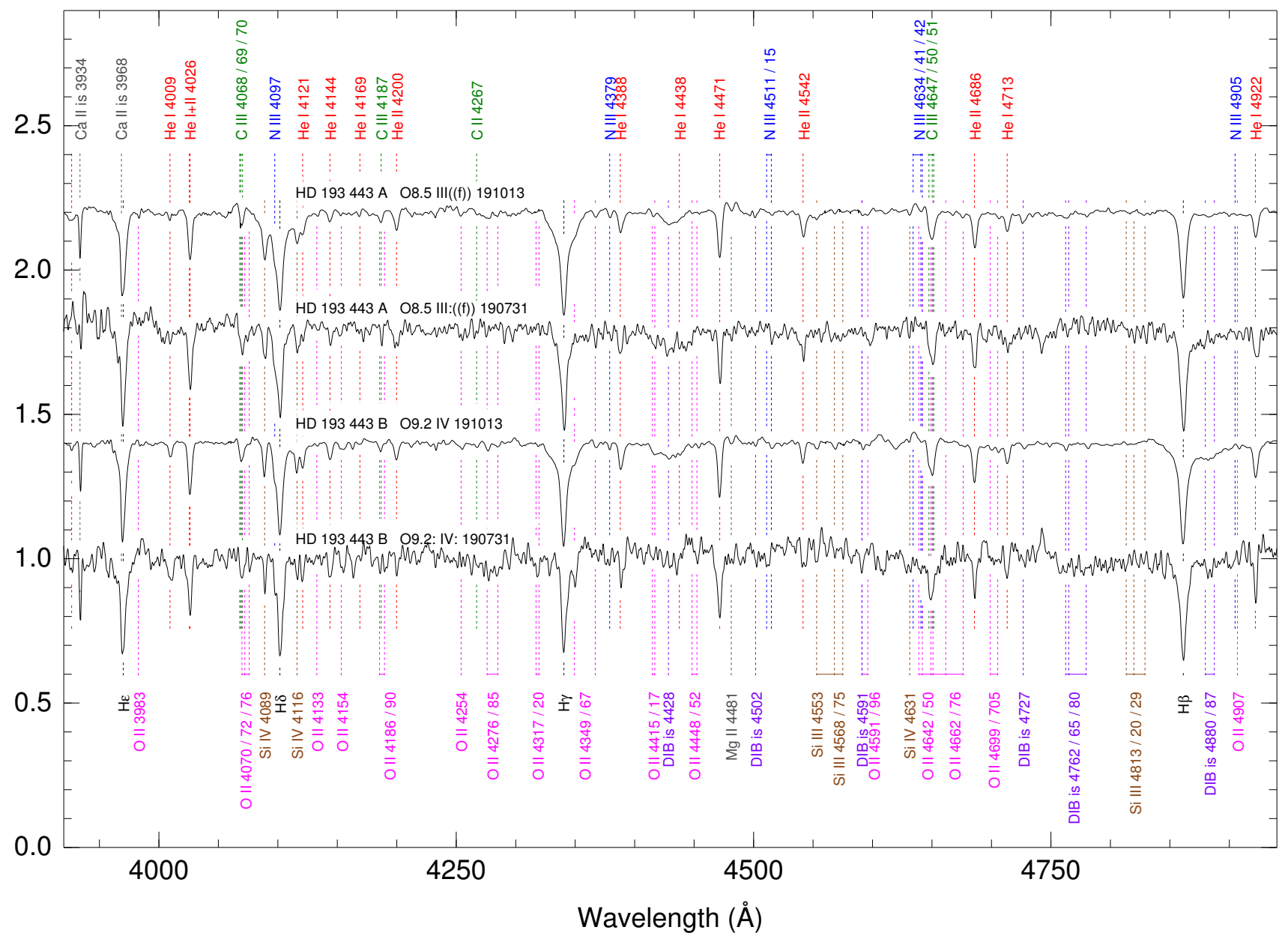

Fig. 17. Rectified spectrograms for HD 193443 at the GOSSS spectral resolution of $R \sim 2500$ and on the stellar reference frame. For each spectrogram, the name, spectral type, and evening date (YYMMDD) are shown. The 191013 data were obtained with STIS/HST and the 190731 with WHT using lucky spectroscopy. Main atomic and ISM lines are indicated.

Table 6. Gaia DR2 astrometric data for nine components of the [KPS2012] MWSC 3335 cluster (not including HD 194649 A,B, for which no Gaia DR2 parallax or proper motions are available) with compatible parallaxes and proper motions and their aggregate results.

\begin{tabular}{lccc}
\hline \hline Gaia DR2 ID & $\begin{array}{c}\varpi \\
\text { (mas) }\end{array}$ & $\begin{array}{c}\mu_{\alpha *} \\
\text { (mas/a) }\end{array}$ & $\begin{array}{c}\mu_{\delta} \\
\text { (mas/a) }\end{array}$ \\
\hline 2067482773947102208 & $0.6009 \pm 0.0269$ & $-3.140 \pm 0.045$ & $-4.242 \pm 0.041$ \\
2067483044525974784 & $0.6250 \pm 0.0308$ & $-3.211 \pm 0.053$ & $-4.134 \pm 0.045$ \\
2067482602148428288 & $0.5542 \pm 0.0264$ & $-3.166 \pm 0.043$ & $-4.118 \pm 0.043$ \\
2067482705227636480 & $0.5593 \pm 0.0240$ & $-3.142 \pm 0.039$ & $-4.180 \pm 0.036$ \\
2067482945745805312 & $0.5753 \pm 0.0168$ & $-3.300 \pm 0.027$ & $-4.409 \pm 0.029$ \\
2067482735291629056 & $0.6065 \pm 0.0245$ & $-3.445 \pm 0.034$ & $-3.811 \pm 0.048$ \\
2067482877026323712 & $0.5757 \pm 0.0189$ & $-2.890 \pm 0.035$ & $-4.450 \pm 0.029$ \\
2067482670867892352 & $0.5944 \pm 0.0194$ & $-3.254 \pm 0.035$ & $-4.293 \pm 0.029$ \\
2067482945745805440 & $0.5826 \pm 0.0230$ & $-3.040 \pm 0.039$ & $-4.181 \pm 0.034$ \\
\hline & $0.5852 \pm 0.0413$ & $-3.177 \pm 0.065$ & $-4.036 \pm 0.065$ \\
\hline
\end{tabular}

Notes. The aggregate results use external uncertainties and include the spatial covariance terms of Lindegren et al. (2018).

near quadrature (when the lucky spectra were obtained), but the two mid-O stars (one in A and one in B) are merged into a single component in velocity. The spectral type of the primary in the merged data (O6) is an intermediate one between the two real types (O5.5 and O7). At the same time, the velocity amplitude of the primary in the SB2 in the merged data is lower than the real value: when we measure the velocity difference between the two components in the merged data, we obtain $\sim 275 \mathrm{~km} \mathrm{~s}^{-1}$, but if we do it in the separated spectrum, we derive $\sim 325 \mathrm{~km} \mathrm{~s}^{-1}$ (at $R \sim 2500$ resolution, individual uncertainties for components well resolved in velocity are $10-15 \mathrm{~km} \mathrm{~s}^{-1}$ ). According to Mahy et al. (2013), $K_{1}=76.3 \mathrm{~km} \mathrm{~s}^{-1}$ and $K_{2}=192.1 \mathrm{~km} \mathrm{~s}^{-1}$ with a circular orbit, which agrees reasonably well with our measurement in the merged spectrum. However, this means that the true value for $K_{1}$ is likely to be closer to $125 \mathrm{~km} \mathrm{~s}^{-1}$. The importance of this result is that it changes the mass ratio from $\sim 2.5$ to $\sim 1.5$. The first value is hard to reconcile with what is expected for an $\mathrm{O} 6 \mathrm{~V}$ and an $09.5 \mathrm{~V}$ star, but the second value is reasonable for an $\mathrm{O} 5.5 \mathrm{~V}$ and an $\mathrm{O} 9.5 \mathrm{~V}$ star. In the future we plan to obtain further data and derive a new orbit within the MONOS project. Finally, the measured 0.95 mag difference between A and B is consistent with the expected value for the derived spectral classifications.

\subsection{HD 191201 A,B, another visual binary with an SB2 component}

This system was included in MONOS I, where we presented spatially unresolved spectroscopy and lucky imaging results. It is a pair with $\Delta m=1.8 \mathrm{mag}$ and $d=0$ "'98. Similarly to HD 194649 , it includes a spectroscopic binary with an $8.333832 \mathrm{~d}$ period 


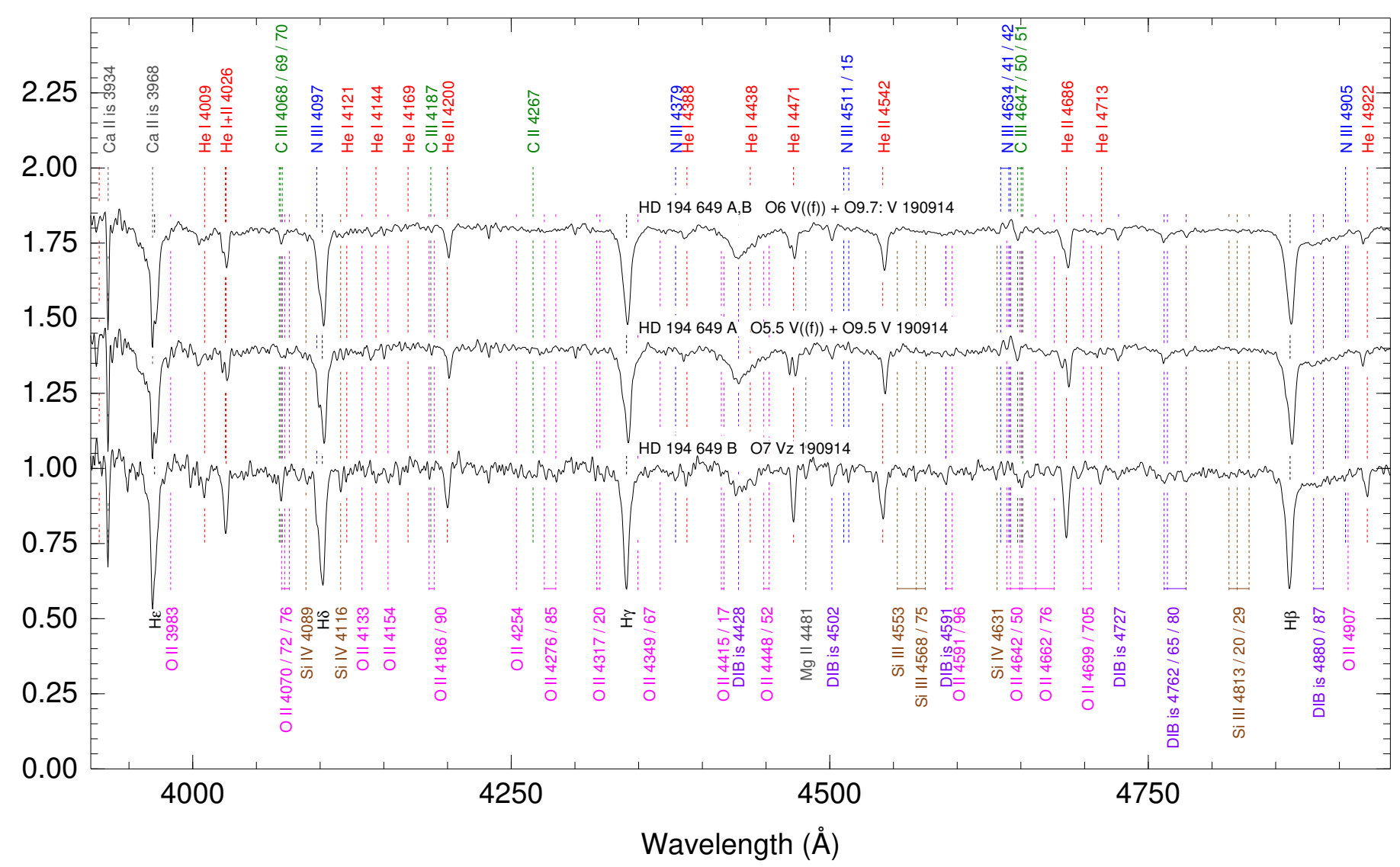

Fig. 18. Rectified spectrograms for HD 194649 at the GOSSS spectral resolution of $R \sim 2500$ and on the heliocentric reference frame. For each spectrogram, the name, spectral type, and evening date (YYMMDD) are shown. The top spectrogram is the weighted combination of the two components for the 190914 epoch. Main atomic and ISM lines are indicated.

(Stickland \& Lloyd 2001), and for this system, it is clear that it resides in the A component. In GOSSS I we classified the A component as O9.5 III + B0 IV and the B component as O9.7 III. HD 191201 A,B has a single entry in Gaia DR2 but with a poor RUWE that does not allow us to use its astrometry to calculate a distance. The WDS catalog lists a C component for this multiple system that does have good-quality astrometry in Gaia DR2. We use it to derive a distance of $2.02_{-0.11}^{+0.13} \mathrm{kpc}$.

We easily resolve the two components of HD 191201 with lucky spectroscopy (Fig. 19). The spectral classification for A is O9.5 III + B0 V, identical to that of GOSSS I, except for the lower luminosity class of the spectroscopic secondary. The spectral classification for B is $09.7 \mathrm{~V}$, downgraded in luminosity class from III with respect to GOSSS I. The difference is likely caused by the lower contamination in the lucky-spectroscopy data compared to the regular long-slit spectroscopy used in the original paper, but there are two additional factors to consider. The first is that since GOSSS I, we have decided to give more weight to the $\mathrm{Si} / \mathrm{He}$ criterion for the luminosity classification of late-type $\mathrm{O}$ stars to the detriment of the $\mathrm{He} / \mathrm{He}$ criterion because the former criterion is less sensitive to the effect of hidden binaries. The second factor is that HD $191201 \mathrm{~B}$ appears to be an object with a very low $v \sin i$. In any case, the change in luminosity classification yields a much better agreement between the expected and the measured $\Delta m$ between $\mathrm{A}$ and $\mathrm{B}$. The spectral classification for the merged spectrum is $\mathrm{O} 9.5 \mathrm{III}+\mathrm{O} 9.7 \mathrm{~V}$, and we see a similar effect as was described for HD 194649: the velocity difference between the two spectroscopic components is larger in the separated spectrum $\left(\sim 300 \mathrm{~km} \mathrm{~s}^{-1}\right.$, obtained near quadrature) than in the merged spectrum $\left(\sim 225 \mathrm{~km} \mathrm{~s}^{-1}\right)$. HD 191201 will also be studied in a future MONOS paper using LiLiMaRlin data.

\section{Summary and future work}

We presented spatially resolved intermediate-resolution blue-violet spectroscopy of 19 massive close binaries. The combined sample of LS I and this paper contains 23 systems with angular separations between 0 ". 138 and 6 ". 19 and physical planeof-the-sky separations between $100 \mathrm{AU}$ and $8000 \mathrm{AU}$. The eight most relevant results are:

1. For two stars (FN CMa B and 6 Cas B) that were previously identified as $\mathrm{O}$ stars based on spatially unresolved data, we obtain for the first time spatially disentangled spectra and derive accurate spectral classifications. In both cases the companion is a more evolved, visually brighter supergiant, of $\mathrm{B}$ type in the first and of A type in the second case. We note that 6 Cas is the only known Galactic case of an A supergiant $+\mathrm{O}$ binary.

2. We determined that two companions to massive stars ( $\alpha$ Sco B and HD 164492 B) are fast rotators of B2 spectral type. In both cases the large $v \sin i$ likely contributed to their misidentification in the literature, in the first case as a B star of a later subtype, and in the second case as an A supergiant. We also note the tendency that we reported in LS I and mentioned for some further cases in STIS I of the visual companions of massive stars to be fast rotators of B spectral type. 


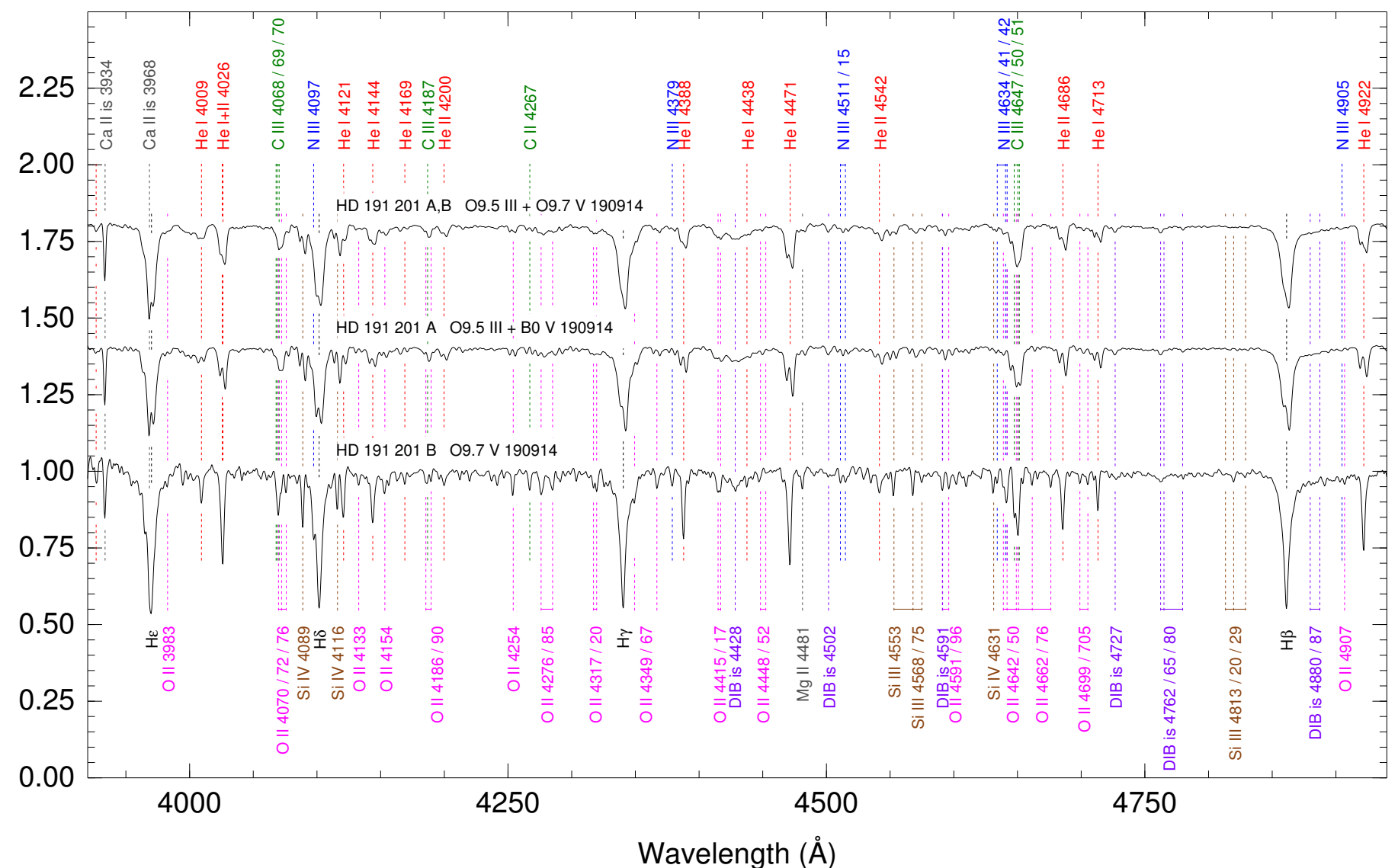

Fig. 19. Rectified spectrograms for HD 191201 at the GOSSS spectral resolution of $R \sim 2500$ and on the heliocentric reference frame. For each spectrogram, the name, spectral type, and evening date (YYMMDD) are shown. The top spectrogram is the weighted combination of the two components for the 190914 epoch. Main atomic and ISM lines are indicated.

3. We have extended the technique of lucky spectroscopy to include three systems ( $\alpha$ Sco A,B, CS Cam A,B, and HD 164492 A,B) with extreme brightness differences (more than $3.5 \mathrm{mag}$ ).

4. Using data from an occasion with exceptional seeing, we were able to spatially separate HD 193443 A,B, a very close system $(d=0$ '.138) that we previously also successfully separated with STIS. The resulting spectra, however, are significantly noisier than the STIS equivalents, indicating that we have reached the limit of the possibilities of lucky spectroscopy (at least with our current setup) for objects with small magnitude differences.

5. We have extended the range of the technique to objects much fainter than those in LS I (down to primaries with $B=11 \mathrm{mag}$ ). We were able to do so in part by using a different CCD that has a better duty cycle. This allows us to stay on target $98 \%$ of the time as opposed to just $7 \%$.

6. In LS I the stars had relatively similar spectral types (mid-O to early-B). Here we expanded the technique to demonstrate that it works with primaries of other spectral types and with systems that differ greatly between primary and secondary. In this way, we have observed as primaries an A supergiant (6 Cas A), a WR star (HD $219460 \mathrm{~B}=\mathrm{WR} 157$ ), and an $\mathrm{M}$ supergiant ( $\alpha$ Sco A). The secondaries in these cases are late-O to early-B stars.

7. Three of the objects in our sample have surprising identities. HD 51756 B and BD +60 544 are two bright stars identified as O-type for the first time, and HD $8768 \mathrm{~A}$ is a new member of the rare OC category. In the three cases the identification was possible because we were able to eliminate the contamination from a nearby companion with lucky spectroscopy.

8. For four of the visual systems in which one of the components is an SB2 ( $\sigma$ Ori, HD 219460, HD 194649, and HD 191201 ), we identified the doubled-lined components and provided the spectral classifications. For HD $219460 \mathrm{~A}$ it is the first time the SB2 nature is noted, and for HD 194649 we identified the A component as the SB2 for the first time. For another seven systems (FN CMa, $\sigma$ Sco, HD 51756, HD 218 195, HD 17 520, HD 24 431, and HD 164 492), we detected signs of spectroscopic binarity in the LiLiMaRlin data.

We plot in Fig. 20 the magnitude difference as a function of separation for the systems in LS I, STIS I, and this paper. The boundaries for the regions accessible for the two methods are just empirical approximations based on the existing data. In particular, the capabilities of lucky spectroscopy depend on the seeing at the moment of the observation: a star that is not spatially resolved today may be resolved tomorrow with the same setup. Moreover, objects near the boundary may be spatially resolved but at the cost of a lower $\mathrm{S} / \mathrm{N}$ because of the noise that is produced by the separation process. In this way, the spectrum of $\sigma \mathrm{Sco} \mathrm{Ab}$ in this paper is noisier than that of HD 168021 because both have similar separations, but the first has a larger $\Delta B$. Similarly, the lucky spectra for HD 193443 A,B were separated, but at the cost of a poor $\mathrm{S} / \mathrm{N}$ because the system lies at the limit of what can be done with lucky spectroscopy and the observation took place with exceptional seeing (the spectra were easily separated with STIS with good $\mathrm{S} / \mathrm{N}$ ). The other object that was 


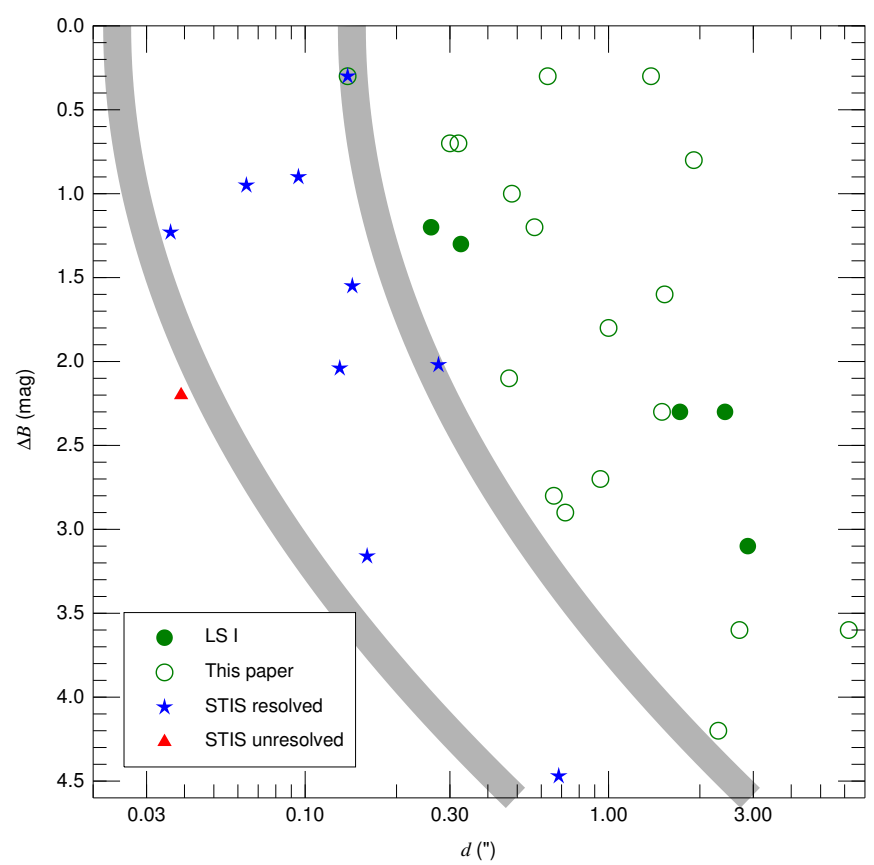

Fig. 20. Binary systems resolved using lucky spectroscopy with WHT/ISIS (in LS I and here) and HST/STIS and unresolved systems using HST/STIS in the $d-\Delta B$ (separation- $B$ magnitude difference) plane. The gray curves mark the tentative empirical boundaries between the regions in the plane accessible with different techniques: the left one is for HST/STIS and the right one for lucky spectroscopy with WHT/ISIS. This is an updated version of Fig. 4 in STIS I.

spatially resolved by STIS lies at the limit accessible by lucky spectroscopy. It is shown in Fig. 20 and is HD $16429 \mathrm{Aa}, \mathrm{Ab}$. We attempted to separate this system twice with lucky spectroscopy, but the conditions were not optimal and we failed.

For the future we plan to obtain additional lucky spectroscopy along four different lines:

1. Observe additional objects using the same configurations as in the first two papers.

2. Obtain observations at higher spectral resolution to better study spectroscopic binaries in hierarchical systems.

3. Extend the technique to longer wavelengths, where there are fewer spectral lines of interest, but where lucky spectroscopy should work better and allow to disentangle systems with smaller separations and/or higher extinctions.

4. Attempt the technique with other telescopes.

Acknowledgements. J.M.A., C.F., A.S., M.P.G., and G.H. acknowledge support from the Spanish Government Ministerio de Ciencia through grant PGC2018-095049-B-C22. R.H.B. acknowledges support from the ESAC Faculty Visitor Program. I.N. and S.S.-D. acknowledge support from the Spanish Government Ministerio de Ciencia through grant PGC2018-093741-B-C21/22 (MICIU/AEI/FEDER, UE). S.S.-D. also acknowledges funding from the Spanish Government Ministerio de Ciencia through grants SEV 2015-0548 and CEX2019-000920-S, and from the Canarian Agency for Research, Innovation and Information Society (ACIISI), of the Canary Islands Government, and the European Regional Development Fund (ERDF), under grant with reference ProID2017010115. This paper is based on (a) lucky (and regular longslit) spectroscopy obtained with the $4.2 \mathrm{~m}$ William Herschel Telescope (WHT) at the Observatorio del Roque de los Muchachos (ORM) on the island of La Palma, Spain; (b) lucky imaging obtained with the $2.2 \mathrm{~m}$ Telescope at the Centro Astronómico Hispano en Andalucía (CAHA) in Almería, Spain; (c) IFU spectroscopy obtained with the $2 \mathrm{~m}$ Liverpool Telescope (LT) at the Observatorio del Roque de los Muchachos (ORM) on the island of La Palma, Spain as part of GOSSS; (d) long-slit spectroscopy obtained with the 2.5 duPont Telescope at the Observatorio de Las Campanas (LCO) in Chile; and (e) high-resolution échelle spectroscopy from the LiLiMaRlin project obtained with a variety of spectrographs: HERMES at the $1.2 \mathrm{~m}$ Mercator Telescope (MT) at the Observatorio del Roque de los Muchachos (ORM) on the island of La Palma, Spain; ELODIE at the $1.93 \mathrm{~m}$ Observatoire de Haute-Provence (OHP) Telescope, France; FEROS at the $2.2 \mathrm{~m}$ Telescope of the Observatorio de La Silla in Chile; CAFÉ at the $2.2 \mathrm{~m}$ Centro Astronómico Hispano en Andalucía (CAHA) Telescope, Almería, Spain; FIES at the 2.5 Nordic Optical Telescope (NOT) at the Observatorio del Roque de los Muchachos (ORM) on the island of La Palma, Spain; and UVES at the $8.2 \mathrm{~m}$ Kueyen Telescope at the Observatorio Paranal in Chile. Some of the MT and NOT data were obtained from the IACOB spectroscopic database (Simón-Díaz et al. 2011b,a, 2015b). This paper has made use of data from the European Space Agency (ESA) mission Gaia (https://www.cosmos.esa.int/gaia), processed by the Gaia Data Processing and Analysis Consortium (DPAC, https://www.cosmos.esa.int/ web/gaia/dpac/consortium). Funding for the DPAC has been provided by national institutions, in particular the institutions participating in the Gaia Multilateral Agreement. This paper has also made use of the Washington Double Star (WDS) catalog (Mason et al. 2001) and the Skiff (2014) catalog of spectral classifications. The authors would like to thank the personnel of the WHT, CAHA, LT, LCO, MT, La Silla, and NOT observatories for their support and hospitality throughout the years. We dedicate this paper to our deceased colleagues, Virpi S. Niemelä and Nolan R. Walborn, who they surely would have enjoyed having access to data like the ones presented here.

\section{References}

Adams, W. S., \& Joy, A. H. 1921, PASP, 33, 206

Arias, J. I., Walborn, N. R., Simón Díaz, S., et al. 2016, AJ, 152, 31 Aydin, C. 1979, Ap\&SS, 64, 481

Beavers, W. I., \& Cook, D. B. 1980, ApJS, 44, 489

Berlanas, S. R., Herrero, A., Comerón, F., et al. 2020, A\&A, 642, A168

Cami, J., Sonnentrucker, P., Ehrenfreund, P., \& Foing, B. H. 1997, A\&A, 326, 822

Campillay, A. R., Arias, J. I., Barbá, R. H., et al. 2019, MNRAS, 484, 2137

Corbally, C. J. 1984, ApJS, 55, 657

Dorda, R., Negueruela, I., González-Fernández, C., \& Marco, A. 2018, A\&A, 618, A137

Finsen, W. S. 1956, Circ. Union Obs. Johannesburg, 115, 259

Gahm, G. F., Ahlin, P., \& Lindroos, K. P. 1983, A\&AS, 51, 143

Garrison, R. F. 1967, ApJ, 147, 1003

Garrison, R. F., Hiltner, W. A., \& Schild, R. E. 1977, ApJS, 35, 111

González, J. F., Hubrig, S., Przybilla, N., et al. 2017, MNRAS, 467, 437

Hardorp, J., Rohlfs, K., Slettebak, A., \& Stock, J. 1959, Hamburger Sternw

(Warner \& Swasey Obs.), C01

Hiltner, W. A. 1956, ApJS, 2, 389

Hiltner, W. A., Garrison, R. F., \& Schild, R. E. 1969, ApJ, 157, 313

Holgado, G., Simón-Díaz, S., Barbá, R. H., et al. 2018, A\&A, 613, A65

Holgado, G., Simón-Díaz, S., Haemmerlé, L., et al. 2020, A\&A, 638, A157

Johnson, H. L., \& Morgan, W. W. 1953, ApJ, 117, 313

Keenan, P. C., \& McNeil, R. C. 1989, ApJS, 71, 245

Kharchenko, N. V., Piskunov, A. E., Schilbach, E., Röser, S., \& Scholz, R.-D. 2013, A\&A, 558, A53

Krełowski, J., Schmidt, M., \& Snow, T. P. 1997, PASP, 109, 1135

Law, N. M., Mackay, C. D., \& Baldwin, J. E. 2006, A\&A, 446, 739

Lesh, J. R. 1968, ApJS, 17, 371

Lindegren, L., Hern'andez, J., Bombrun, A., et al. 2018, https: //www. cosmos.esa.int/documents/29201/1770596/Lindegren_ GaiaDR2_Astrometry_extended.pdf

Mahy, L., Rauw, G., De Becker, M., Eenens, P., \& Flores, C. A. 2013, A\&A, 550, A27

Mahy, L., Rauw, G., De Becker, M., Eenens, P., \& Flores, C. A. 2015, A\&A, 577, A23

Maíz Apellániz, J. 2001, AJ, 121, 2737

Maíz Apellániz, J. 2005, in The Three-Dimensional Universe with Gaia, eds. C. Turon, K. S. O'Flaherty, \& M. A. C. Perryman, ESA SP, 576, 179

Maíz Apellániz, J. 2010, A\&A, 518, A1

Maíz Apellániz, J. 2015, Mem. Soc. Astron. It., 86, 553

Maíz Apellániz, J. 2019, A\&A, 630, A119

Maíz Apellániz, J., \& Barbá, R. H. 2018, A\&A, 613, A9

Maíz Apellániz, J., \& Barbá, R. H. 2020, A\&A, 636, A28

Maíz Apellániz, J., Alfaro, E. J., \& Sota, A. 2008, ArXiv e-prints [arXiv:0804.2553]

Maíz Apellániz, J., Sota, A., Walborn, N. R., et al. 2011, in The Galactic O-star spectroscopic survey (GOSSS), HSA, 6, 467

Maíz Apellániz, J., Sota, A., Arias, J. I., et al. 2016, ApJS, 224, 4

Maíz Apellániz, J., Barbá, R. H., Simón-Díaz, S., et al. 2018, A\&A, 615, A161 
Maíz Apellániz, J., Trigueros Páez, E., Jiménez Martínez, I., et al. 2019a, in LiLiMaRlin, a Library of Libraries of Massive-Star High-Resolution Spectra with applications to OWN, MONOS, and CollDIBs, HSA, 10, 420

Maíz Apellániz, J., Trigueros Páez, E., Negueruela, I., et al. 2019b, A\&A, 626, A20

Maíz Apellániz, J., Crespo Bellido, P., Barbá, R. H., Fernández Aranda, R., \& Sota, A. 2020a, A\&A, 643, A138

Maíz Apellániz, J., Barbá, R. H., Caballero, J. A., Bohlin, R. C., \& Fariña, C. 2020b, MNRAS, 2498

Mason, B. D., Gies, D. R., Hartkopf, W. I., et al. 1998, AJ, 115, 821

Mason, B. D., Wycoff, G. L., Hartkopf, W. I., Douglass, G. G., \& Worley, C. E. 2001, AJ, 122, 3466

Morgan, W. W., \& Roman, N. G. 1950, ApJ, 112, 362

Morgan, W. W., \& Keenan, P. C. 1973, ARA\&A, 11, 29

Morgan, W. W., Whitford, A. E., \& Code, A. D. 1953, ApJ, 118, 318

Morgan, W. W., Code, A. D., \& Whitford, A. E. 1955, ApJS, 2, 41

North, J. R., Davis, J., Tuthill, P. G., Tango, W. J., \& Robertson, J. G. 2007, MNRAS, 380, 1276

Pantaleoni González, M., Maíz Apellániz, J., Barbá, R. H., \& Negueruela, I. 2020, RNAAS, 4, 12

Reimers, D., Hagen, H.-J., Baade, R., \& Braun, K. 2008, A\&A, 491, 229

Rivinius, T., Stahl, O., Štefl, S., et al. 2011, in The (B0+?)+O6 System FN CMa: a Case for Tidal-pulsational Interaction?, IAUS, 272, 543

Roman-Lopes, A., Román-Zúñiga, C., Tapia, M., et al. 2018, ApJ, 855, 68

Rydstrom, B. A. 1978, A\&AS, 32, 25

Sana, H., Le Bouquin, J.-B., Lacour, S., et al. 2014, ApJS, 215, 15
Simón-Díaz, S., \& Herrero, A. 2014, A\&A, 562, A135

Simón-Díaz, S., Castro, N., García, M., \& Herrero, A. 2011a, in The IACOB Spectroscopic Database of Galactic OB Stars, IAUS, 272, 310

Simón-Díaz, S., Garcia, M., Herrero, A., Maíz Apellániz, J., \& Negueruela, I 2011b, Stellar Clusters \& Associations: A RIA Workshop on Gaia, 255

Simón-Díaz, S., Herrero, A., Sabín-Sanjulián, C., et al. 2014, A\&A, 570, L6

Simón-Díaz, S., Caballero, J. A., Lorenzo, J., et al. 2015a, ApJ, 799, 169

Simón-Díaz, S., Negueruela, I., Maíz Apellániz, J., et al. 2015b, in The IACOB Spectroscopic Database: Recent Updates and First Data Release, HSA, 8, 576 Simón-Díaz, S., Godart, M., Castro, N., et al. 2017, A\&A, 597, A22

Simón-Díaz, S., Britavskiy, N., Castro, N., Holgado, G., \& de Burgos, A. 2020, A\&A, submitted

Skiff, B. A. 2014, VizieR Online Data Catalog: II/307

Smith, L. F., Shara, M. M., \& Moffat, A. F. J. 1996, MNRAS, 281, 163

Sota, A., Maíz Apellániz, J., Walborn, N. R., et al. 2011, ApJS, 193, 24

Sota, A., Maíz Apellániz, J., Morrell, N. I., et al. 2014, ApJS, 211, 10

Stickland, D. J., \& Lloyd, C. 2001, Observatory, 121, 1

Stone, S. N., \& Struve, O. 1954, PASP, 66, 191

Swings, J. P., \& Preston, G. W. 1978, ApJ, 220, 883

Talavera, A., \& Gómez de Castro, A. I. 1987, A\&A, 181, 300

Turner, D. G., Moffat, A. F. J., Lamontagne, R., \& Maitzen, H. M. 1983, AJ, 88, 1199

Turner, N. H., ten Brummelaar, T. A., Roberts, L. C., et al. 2008, AJ, 136, 554

Wade, G. A., Shultz, M., Sikora, J., et al. 2017, MNRAS, 465, 2517

Walborn, N. R. 1972, AJ, 77, 312

Walborn, N. R. 1973, AJ, 78, 1067

Ward, J. L., Kruijssen, J. M. D., \& Rix, H.-W. 2020, MNRAS, 495, 663 